\title{
3 Research Square

\section{Dynamic characteristics of a gear system with double-teeth spalling fault and its fault feature analysis}

\section{Luojie Shi}

Zhejiang University of Technology

Juan Wen

Zhejiang University of Technology

Baisong Pan ( $\sim$ panbsz@zjut.edu.cn )

Zhejiang University of Technology

\section{Yongyong Xiang}

Zhejiang University of Technology

\section{Qi Zhang}

Zhejiang Shuanghuan Driveline Co., Ltd

\section{Congkai Lin}

Zhejiang University of Technology

\section{Original Article}

Keywords: Gear systems, Double-teeth spalling fault, Fault feature analysis, Variational mode decomposition, Fast kurtogram

Posted Date: May 5th, 2020

DOI: https://doi.org/10.21203/rs.3.rs-26058/v1

License: (c) (1) This work is licensed under a Creative Commons Attribution 4.0 International License.

Read Full License

Version of Record: A version of this preprint was published at Applied Sciences on October 11th, 2020. See the published version at https://doi.org/10.3390/app10207058. 


\section{Title page}

\section{Dynamic characteristics of a gear system with double-teeth spalling fault and its fault feature analysis}

Luo-Jie Shi, born in 1994, is currently a master candidate at College of Mechanical Engineering, Zhejiang University of Technology, China. His main research interests include machinery fault diagnostics, and reliability analysis.

E-mail: luojieshi@zjut.edu.cn

Juan Wen, born in 1990, is currently an assistant professor at College of Mechanical Engineering, Zhejiang University of Technology, China. Her main research interests include machinery condition monitoring, intelligent fault diagnosis, and remaining useful life prediction.

E-mail: juanwen@zjut.edu.cn

Bai-Song Pan, born in 1968, is currently an professor at College of Mechanical Engineering, Zhejiang University of Technology, China. His main research interests include reliability design, and machinery fault diagnostics.

E-mail: panbsz@zjut.edu.cn

Yong-Yong Xiang, born in 1991, is currently a PhD candidate at College of Mechanical Engineering, Zhejiang University of Technology, China. His main research interests include uncertainty analysis, and model validation and calculation.

E-mail: xiangyyong@126.com

Qi Zhang, born in 1984, is currently a senior engineer at Zhejiang Shuanghuan Driveline Co., Ltd., Zhejiang Province, China. His main research interests include mechanical system design \& NVH testing.

E-mail: zhangqi@gearsnet.com

Cong-Kai Lin, born in 1995, is currently a master candidate at College of Mechanical Engineering, Zhejiang University of Technology, China. His main research interests include reliability analysis method application and algorithm reaearch.

E-mail: lincongkai7980@163.com

\section{Corresponding author: Bai-Song Pan E-mail: panbsz@zjut.edu.cn}




\title{
Dynamic characteristics of a gear system with double-teeth spalling fault and its fault feature analysis
}

\author{
Luo-jie Shi ${ }^{1,2}$ • Juan Wen ${ }^{1,2}$ • Bai-Song Pan ${ }^{1,2}$ • Yong-Yong Xiang ${ }^{1,2}$ • Qi Zhang ${ }^{3}$ Cong-Kai Lin ${ }^{1,2}$
}

Received June xx, 201x; revised February xx, 201x; accepted March xx, 201x

(c) Chinese Mechanical Engineering Society and Springer-Verlag Berlin Heidelberg 2017

\begin{abstract}
Gear fault diagnosis has been a vital technology to enhance the reliability and reduce the maintenance cost of gear systems. Tooth spalling is one of the most destructive surface failure models of the gear faults. Revealing the dynamic characteristics of a gear system with spalling fault and extracting the fault feature are the premise and basis for effective fault diagnosis. Previous studies have mainly concentrated on the spalling damage on a single gear tooth, but the spalling distributed over double teeth which usually occurs in practical engineering problems is rarely reported. To remedy this deficiency, this paper constructs a new dynamical model of a gear system with double-teeth spalling fault and validates this model with various experimental tests. The dynamic characteristics of gear systems are obtained by considering the excitations induced by the number of spalling teeth, the relative position of two faulty teeth, and the rotational speed. The method based on the Variational Mode Decomposition (VMD) and the Fast Kurtogram (FK) is proposed to extract the features of the double-teeth spalling fault. First, the raw signal is decomposed into a set of Intrinsic Mode Functions (IMFs) by applying the VMD, and the IMFs with strong correlation are summed as a reconstructed signal. The reconstructed signal is then filtered by an optimal band-pass filter based on the FK. Combined with envelope spectrum analysis, the feature extraction ability of the proposed method is compared with that of the original FK method and the

Bai-Song Pan

panbsz@zjut.edu.cn

1 College of Mechanical Engineering, Zhejiang University of Technology, Hangzhou 310023, China

2 Key Laboratory of Special Purpose Equipment and Advanced Processing Technology, Ministry of Education and Zhejiang Province, Zhejiang University of Technology, Hangzhou 310023, China

3 Shuanghuan Driveline Co., Ltd., Electrical and Industrial Zone, Yuhuan County, Zhejiang, 317600, China
\end{abstract}

method based on the Empirical Mode Decomposition and the FK, respectively. The results indicate that the proposed dynamic model and fault feature extraction method can provide a theoretical basis for spalling defect diagnosis of gear systems.

Keywords: Gear systems • Double-teeth spalling fault • Fault feature analysis - Variational mode decomposition • Fast kurtogram

\section{Introduction}

Gear systems commonly exist in mechanical equipment, such as wind turbines, shearer, and motor vehicle [1]. Due to the excessive load, poor lubrication, and other harsh operating conditions, faults often occur in the gear systems. The faults may generate serious economic losses and even casualties. Specifically, damages (e.g., pitting, cracking, and spalling) on the gear teeth are the main causes of system failure, and they usually impact the dynamic characteristics of gear devices [2-5]. Background noise often occurs in actual fault detection. It is difficult to extract fault feature accurately when background noise involves [6]. Therefore, it is essential to effectively obtain the dynamic characteristics of gear systems and extract the fault feature for identifying faults because they play an important role in improving reliability and reducing maintenance cost of gear systems.

Spalling is one of the most destructive surface failure models of gear faults [7]. Until now, many researchers have modeled the tooth spalling and investigated its effect on dynamic characteristics of the gear systems [8]. According to their researches, tooth spalling was regarded as different shapes (e.g., rectangle, circular, and V-shape), and the influence of the spalling on time-vary mesh stiffness (TVMS) of gear was explored [9-11]. Luo et al. 
[12] proposed a curved bottom shape method to model the geometric features of gear tooth spalls and studied the effect of different shapes and severity conditions time-vary mesh stiffness by finite element analysis. Chen et al. [13] presented a new two-dimensional Gaussian distribution model to depict pitting distribution. In their analysis, the overlaps of the pits and pits in the boundary of the tooth surface were considered in the computation of TVMS. Furthermore, Luo et al. [14] built a dynamic model that includes tooth surface roughness changes and geometric deviations because of the pitting and spalling of gear teeth. This model was validated by comparison with experiments under different rotational speed and fault conditions. The above-mentioned studies only focus on a single tooth spalling fault. In fact, tooth spalling which is caused by excessive local Hertzian contact fatigue stress may distribute over multiple teeth [15-16]. In term of this phenomenon, Liang et al. [17] modeled a gear system with double-teeth spalling fault, and simulated its effect on vibration characteristics. However, in Ref. [17], only the phenomenon that the tooth spalling occurs on two adjacent teeth is considered. According to Ref. [17], this paper will further explore the influence of the relative position of two faulty teeth on the dynamic characteristics of gears.

The accuracy of dynamic model validation results and the validity of the fault feature extraction are susceptible to the background noise caused by the sensor's location (e.g., the bearing pedestal or the gearbox housing). It is of great significance for dynamic model validation and fault diagnosis to highlight the fault feature and eliminate the background noise in the experimental signal. Spectral Kurtosis (SK) which was proposed by Dwyer for detecting non-stationary components to highlight the features in a signal is an effective tool [18]. It can reveal the hidden non-stationary components and indicate their frequency bands by calculating the kurtosis index of each frequency line. Antoni [19] made further efforts on the SK and formally defined it as a fourth-order spectral cumulant. For improving the efficiency and accuracy of the SK calculation, Antoni presented two methods [20-21]. One was Kurtogram based on the Short-Time Fourier Transform, the other was Fast Kurtogram based on the arborescent filter-bank structure. Compared with Kurtogram, the FK has been widely used to highlight the fault feature for mechanical fault diagnosis because of high efficiency [22-23]. Nevertheless, the efficiency of the FK is limited by the complex background noise in the original signal [24]. To tackle the complex background, several signal processing methods such as Wavelet Transform (WT) [25-26], Empirical Mode Decomposition (EMD) [27-32],
Ensemble Empirical Mode Decomposition (EEMD) [33-34], and Local Mean Decomposition (LMD) [35-36] have been applied. However, the treatment effect of these methods is often not satisfactory because of the inherent limitations. The WT method needs to empirically chose wavelet basis function. The EMD method decomposes any signal into a set of intrinsic mode functions (IMFs), but its efficiency is restricted by the problems of end effects and mode mixing. The EEMD method mainly depends on the parameters of white noise. The LMD method is confined by decomposition level. In recent years, a lot of literature has studied a new adaptive signal processing method which is called Variational Mode Decomposition (VMD) [37]. By determining the relevant bands of fault feature and dividing the signal into several IMFs, the VMD can effectively avoid the end effects and mode mixing [38-39]. Zhang et al. [40] proved that the VMD performs better than the EMD. An et al. [41] compared the performance of the VMD, the EMD, and the WT, and proved that the VMD outperforms other methods. Considering the above all, many novel signal processing methods around the VMD have been proposed [42-45]. Based on the advantages of the VMD in denoising, this paper uses the VMD to eliminate the complex background noise in the experimental signal.

Generally, gear system dynamic characteristics and fault feature extraction methods that considers the double-teeth spalling fault have not been well studied by existing researches. To make this gap, a new dynamic model of gear systems considering the double-teeth spalling fault is established in this paper. This dynamic model is validated by various experimental tests, and could supply more realistic dynamic environment for extraction of gear double-teeth fault feature. Then, the dynamic characteristics considering the effect of the number of spalling teeth, the relative position of two faulty teeth, and the rotational speed are analyzed. The method based on the VMD and the FK is used to extract the features of double-teeth spalling fault embedded the strong background noise. The raw signal acquired from gear systems is decomposed into several IMFs by using the VMD, and the IMFs with strong correlation are summed as a reconstructed signal. An optimal band-pass filter based on the FK is applied to filter the reconstructed signal .

The main contributions of this paper are summarized as follows: (1) a new dynamical model which considers double-teeth spalling fault is proposed, and the dynamic characteristics considering the effect of the relative position of two faulty teeth are investigated. This investigation is proposed for the first time in previous 
studies; (2) by integrating the VMD and the FK, a novel fault extraction method for gear fault detection is proposed. The original signal is denoised by using the VMD, which ensures the validity of the FK in fault feature extraction.

The rest of the paper is organized as following. The TVMS calculation method considering the double-teeth spalling fault is introduced in Section 2, and In Section 3, the new dynamical model is proposed. In Section 4, the effectiveness of the dynamic model is validated by analyzing the dynamic characteristics of the gear system and some influencing factors are discussed. In Section 5, the fault extraction method based on the VMD and the FK is detailed. Conclusions are provided in Section 6.

\section{Meshing stiffness calculation of gears}

As shown in Figure 1, the gear meshing transmission is divided into double toothed meshing zone and single toothed meshing zone. The meshing stiffness of the gear will change abruptly when the double toothed meshing zone and single toothed meshing zone alternate.
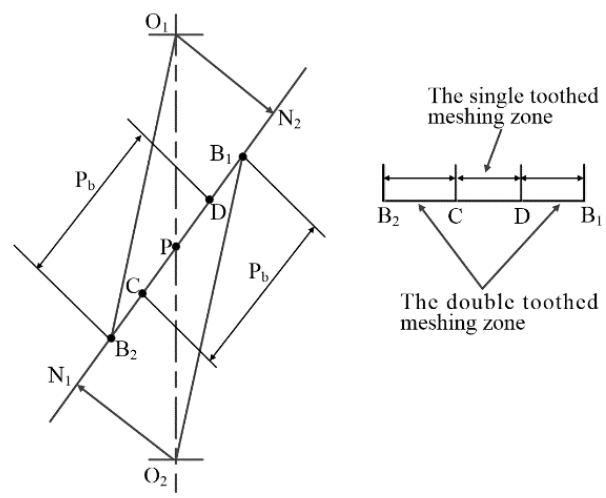

Figure 1 Schematic diagram of gear meshing

\subsection{Meshing stiffness calculation of gears with healthy tooth}

As shown in Figure 2, the gear teeth is divided into $n$ microelements as a cantilever.

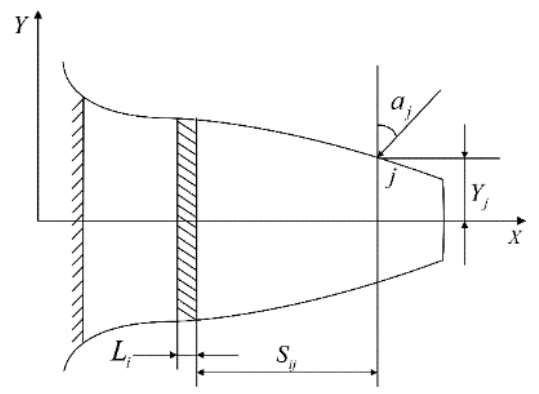

Figure 2 Schematic diagram of a gear tooth cantilever beam
On the basis of the Timoshenko's beam theory [46], the stiffness of the tooth bending, shear and axial compressive are given as

$$
\frac{1}{k_{b j}}=\sum_{i=1}^{n} \frac{1}{E_{e}}\left(\begin{array}{l}
\frac{L_{i}^{3}+3 L_{i}^{2} S_{i j}+3 L_{i} S_{i j}^{2}}{3 I_{j}} \cos ^{2} a_{j}- \\
\frac{L_{i}^{2} Y_{j}+2 L_{i} Y_{j} S_{i j}}{2} \cos a_{j} \sin a_{j}+ \\
\frac{12(1+v) L_{i}}{5 A_{j}} \cos ^{2} a_{j}+\frac{L_{i}}{A_{j}} \sin ^{2} a_{j}
\end{array}\right),
$$

where $L_{i}$ and $a_{j}$ represent the thickness of a microelement and the pressure angle of the contact point $j$, respectively; $S_{i j}$ is the distance between a microelement and the contact point in the $\mathrm{X}$ direction; $Y_{j}$ is the corresponding half tooth thickness; $A_{j}$ and $I_{j}$ denote the cross-sectional areas and area moments of inertia, respectively. The $A_{j}$ and $I_{j}$ could be expressed by Eqs. (2), (3).

$$
\begin{gathered}
A_{j}=2 B Y_{j} \\
I_{j}=\frac{1}{12} B\left(2 Y_{j}\right)^{3}
\end{gathered}
$$

$E_{e}$ represents the effective-Young's modulus which can be expressed as

$$
E_{e}=\left\{\begin{array}{ll}
E & \frac{B}{H_{p}}<5 \\
\frac{E}{1-v^{2}} & \frac{B}{H_{p}} \geq 5
\end{array},\right.
$$

in which, $E$ and $v$ are the Young's modulus and the Poisson's ratio, respectively; $B$ is the teeth width, and $H_{p}$ is the thickness on the pitch circle.

Eq. (5) represents the fillet foundation stiffness according the Muskhelishivili's method [47].

$$
\frac{1}{k_{f j}}=\frac{\cos ^{2} a_{j}}{B E_{e}}\left(L^{*} \frac{u_{f}^{2}}{s_{f}^{2}}+M^{*} \frac{u_{f}}{s_{f}}+P^{*}\left(1+Q^{*} \tan ^{2} a_{j}\right)\right)
$$

The Hertzian contact stiffness is calculated by Eq. (6).

$$
\frac{1}{k_{h j}}=\frac{4\left(1-v^{2}\right)}{\pi B E}
$$


The total structural stiffness of a tooth pair is defined as

$$
k_{p g}=\frac{1}{\frac{1}{k_{h}}+\frac{1}{k_{b p}}+\frac{1}{k_{f p}}+\frac{1}{k_{b g}}+\frac{1}{k_{f g}}}
$$

where $p$ and $g$ are pinion and gear, respectively.

\subsection{Meshing stiffness calculation of gears with tooth spalling}

The calculation method of meshing stiffness of gears with spalling teeth is similar to that of healthy gears. Both of these calculation methods consider the axial compressive stiffness, shear stiffness, bending stiffness, fillet foundation stiffness and Hertzian contact stiffness.
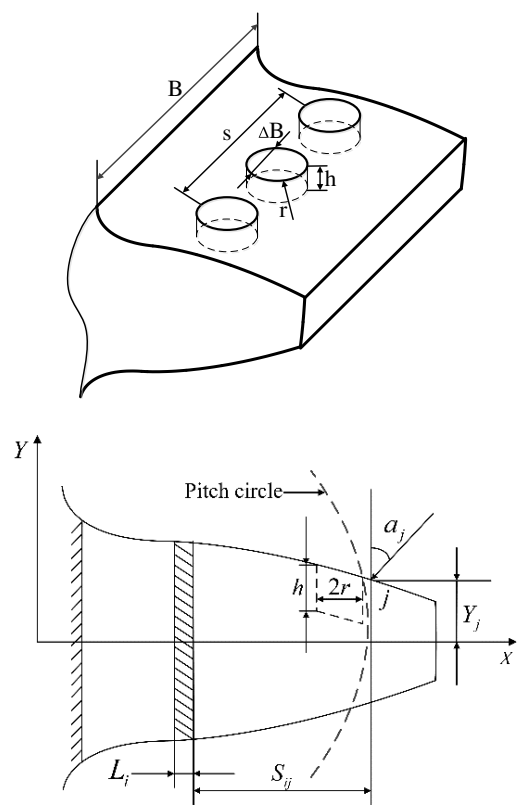

Figure 3 Geometric model of the spalling tooth

In this paper, spalling is molded as a circular shape and occurred near the gear pitch line, as shown in Figure 3. For a gear tooth with spalling, the expressions of $\Delta B, A_{j}$ ' and $I_{j}$, are given as follows:

$$
\begin{gathered}
\Delta B=\sum_{i=1}^{n} 2 \sqrt{r^{2}-\left[(d / 2-r)-s_{i j}\right]^{2}}, \\
A_{j}^{\prime}=2 B Y_{j}-s h \Delta B, \\
I_{j}^{\prime}=I_{j}-\frac{1}{12} s \Delta B h^{3}-\frac{A_{j} \operatorname{sh} \Delta B\left(Y_{j}-h / 2\right)^{2}}{A_{j}^{\prime}},
\end{gathered}
$$

where $\Delta B, A_{j}^{\prime}$ and $I_{j}^{\prime}$ represent the reduction of tooth contact width, area and area moment of inertia of the tooth spalling section, respectively; $r, h$ and $s$ are the radius, depth, and number of spalling points, respectively; $d$ is the diameter of the pitch circle.

\section{Dynamic model of a gear system}

A six-degree of freedom dynamical model considering TVMS, transmission error, and bearing support stiffness is established, in which the changes of TVMS are caused by double-teeth spalling fault, as shown in Figure 4.

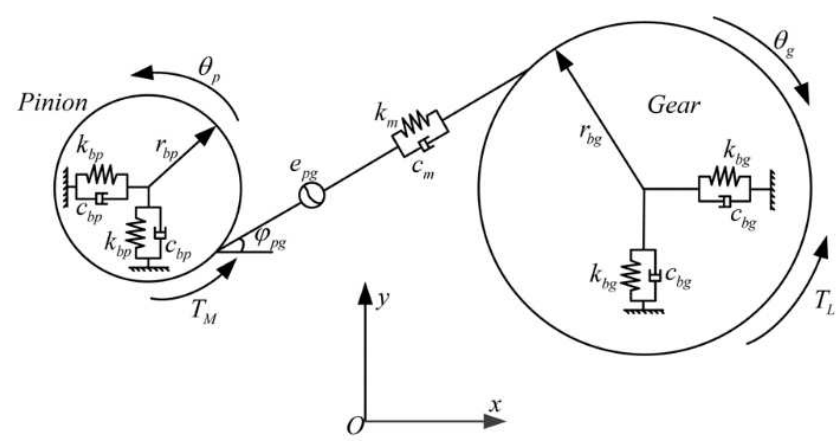

Figure 4 Dynamic model of the 6-DOF gear system

Here are some assumptions: (1) the bearings at both ends of the shaft have the same stiffness and damping; (2) the gearbox casing is a rigid body; (3) the shaft of the pinion and gear are parallel to each other. The equations of the dynamic model are given by Eq. (11).

$$
\left\{\begin{array}{l}
m_{p} \ddot{x}_{p}+c_{b p} \dot{x}_{p}+k_{b p} x_{p}+F_{p g} \cos \varphi_{p g}=0 \\
m_{p} \ddot{y}_{p}+c_{b p} \dot{y}_{p}+k_{b p} y_{p}+F_{p g} \sin \varphi_{p g}=0 \\
I_{p} \ddot{\theta}_{p}+r_{b p} F_{p g}=T_{M} \\
m_{g} \ddot{x}_{g}+c_{b g} \dot{x}_{g}+k_{b g} x_{g}-F_{p g} \cos \varphi_{p g}=0 \\
m_{g} \ddot{y}_{g}+c_{b g} \dot{y}_{g}+k_{b g} y_{g}-F_{p g} \sin \varphi_{p g}=0 \\
I_{g} \ddot{\theta}_{g}-r_{b g} F_{p g}=-T_{L}
\end{array}\right.
$$

$F_{p g}$ denotes the total mesh force, and it is expressed by

$$
\begin{aligned}
F_{p g}=c_{m}\left[\begin{array}{l}
r_{b p} \dot{\theta}_{p}-r_{b g} \dot{\theta}_{g}-\left(\dot{x}_{p}-\dot{x}_{g}\right) \cos \varphi_{p g}- \\
\left(\dot{y}_{p}-\dot{y}_{g}\right) \sin \varphi_{p g}-\dot{e}_{p g}
\end{array}\right]+ \\
k_{m}\left[\begin{array}{l}
r_{b p} \theta_{p}-r_{b g} \theta_{p}-\left(x_{p}-x_{g}\right) \cos \varphi_{p g}- \\
\left(y_{p}-y_{g}\right) \sin \varphi_{p g}-e_{p g}
\end{array}\right],
\end{aligned}
$$

where $r_{b p}$ and $r_{b g}$ are the base circle radius of pinion and 
gear; $I_{p}$ and $I_{g}$ are the mass moments of inertia of pinion and gear; $m_{p}$ and $m_{g}$ are the masses of the pinion and gear; $k_{b p}$ and $k_{b g}$, are the bearing stiffness of the pinion and gear, respectively; $c_{b p}$ and $c_{b g}$ are the bearing damping of the pinion and gear, respectively; $T_{M}$ is the input torque, $T_{L}$ is the load torque; the time-varying meshing damping $c_{m}$ is mentioned in [48]; $e_{p g}$ denotes transmission error. The transmission error considers the short-period error in this paper, which can be obtained:

$$
e_{p g}(t)=\Delta f_{i c}^{\prime} \cos (a) \sin \left(2 \pi f_{m}+\phi\right)
$$

where $a$ is the pressure angle, $f_{m}$ is the mesh frequency of the gear, and $\phi$ is the phase.

According to the definition of $\Delta f^{\prime}{ }_{i c}, \Delta f^{\prime}{ }_{i c}$ can be expressed as:

$$
\Delta f_{i c}^{\prime}=\Delta f_{i p}^{\prime}+\Delta f_{i g}^{\prime} \leq f_{i p}^{\prime}+f_{i g}^{\prime}
$$

where $f^{\prime}{ }_{i p}=\Delta f_{p b p}+\Delta f_{f p}, f^{\prime}{ }_{i g}=\Delta f_{p b g}+\Delta f_{f g}$, in which $\Delta f_{p b p}$ and $\Delta f_{p b g}$ are the pitch deviation of the pinion and gear, respectively; $\Delta f_{f p}$ and $\Delta f_{f g}$ are the profile deviation of the pinion and gear, respectively; as shown in Figure 5.

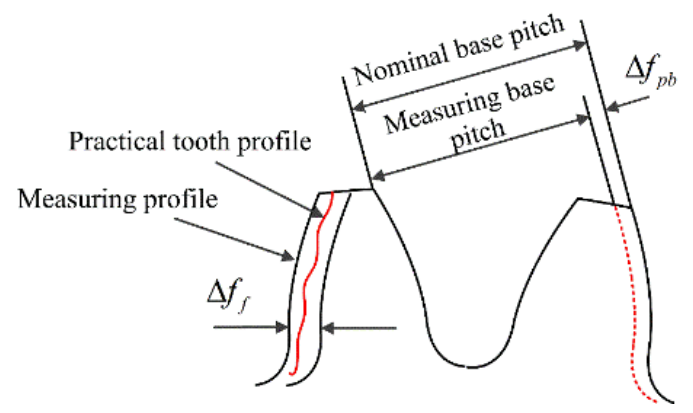

Figure 5 Tooth error and base pitch error[49]

\section{Dynamic simulation and experimental verification}

The simulated vibration signal is compared with the experimental measurement signal in time and frequency domain, and the dynamic simulation results are verified under conditions of different faulty tooth number, relative position of faulty teeth, and rotational speed. The simulation parameters are detailed in Table 1.

The test-rig presented in Figure 6 is the standard FZG test-rig of STRAMA-MPS. This FZG test-rig consists of a controller, a three-phase motor, a test gearbox, and a load applied by a clutch. Table 1 indicated the performance parameters of the bearing and gear. The data acquisition system is B\&K-3560C, and the experimental vibrations signal is measured along the meshing line of the gear teeth by the sensor mounted on the bearing pedestal. The sampling frequency is $65536 \mathrm{~Hz}$, and the sampling time is $1 \mathrm{~s}$. Since the pinion is a faulty gear, the vibration signal $a_{p y}$ of the pinion in the direction of the meshing line is collected.

Table 1 Parameters for the dynamic simulation

\begin{tabular}{lll}
\hline Parameters & Pinion & Gear \\
\hline Number of teeth & 16 & 24 \\
Pressure angle $(\mathrm{deg})$ & \multicolumn{2}{c}{20} \\
Teeth module $(\mathrm{mm})$ & \multicolumn{2}{c}{4.5} \\
Teeth width $(\mathrm{mm})$ & \multicolumn{2}{c}{14} \\
Mass $(\mathrm{kg})$ & 0.676 & 1.084 \\
Mass moment inertia $\left(\mathrm{kg} \cdot \mathrm{m}^{2}\right)$ & 0.000407 & 0.001168 \\
Young's modulus $\left(\mathrm{N} / \mathrm{mm}^{2}\right)$ & $2.06 \mathrm{e} 5$ \\
Poisson's ratio & 0.3 & \\
Bearing damping $(\mathrm{N} \cdot \mathrm{s} / \mathrm{m})$ & $4.86 \mathrm{e} 3$ & $6.16 \mathrm{e} 3$ \\
Bearing stiffness $(\mathrm{N} / \mathrm{m})$ & $3.5 \mathrm{e} 9$ & $3.5 \mathrm{e} 9$ \\
Torque $(\mathrm{N} \cdot \mathrm{m})$ & 23.5 & 35.3 \\
\hline
\end{tabular}

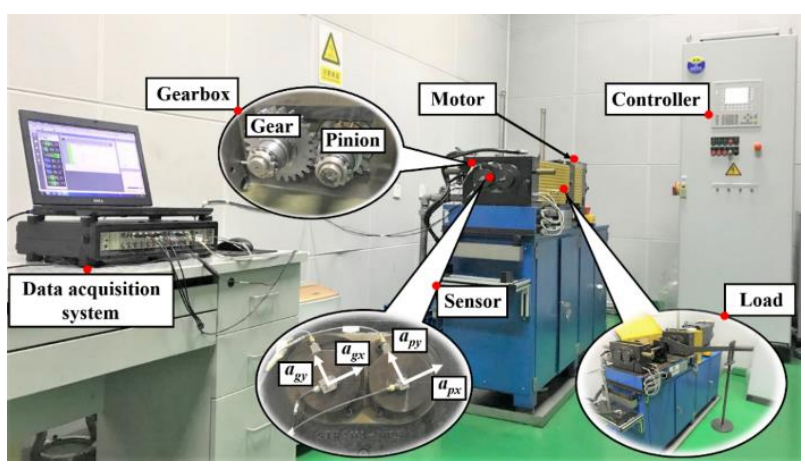

Figure 6 The FZG test-rig

\subsection{Effect of the number of spalling faulty teeth on dynamic characteristics of gears}

In this paper, we assume that the rotational speed of pinion is $n=1500 \mathrm{r} / \mathrm{min}$, the rotational frequency of pinion is $f_{s}=25$ $\mathrm{Hz}$, the rotating period of pinion is $t_{s}=0.04 \mathrm{~s}$, the meshing frequency is $f_{m}=400 \mathrm{~Hz}$, and the meshing period is $t_{m}=0.0025 \mathrm{~s}$. Three types of the spalling faulty tooth number are considered, namely health pinion, pinion with single tooth spalling, and pinion with adjacent double teeth spalling.

Figure 7(a), Figure 8(a), and Figure 9(a) present the failure states of tooth surface spalling, respectively. It can clearly see that the spalling near the pitch line. This paper we set that $r=0.75 \mathrm{~mm}, h=1 \mathrm{~mm}$, and $s=3$, where $r, h$, and $s$ 
are the radius, depth, and numbers of spalling point, respectively. According to the method described in Section 2 , the meshing stiffness is simulated. It can be observed from Figure 7(b) that the meshing stiffness curve is periodically changing during the meshing process. The meshing stiffness decreases when the spalling faulty tooth participates in an engagement. The frequency of meshing stiffness reduction is related to the number of teeth with spalling fault, as shown in Figure 8(b) and Figure 9(b).

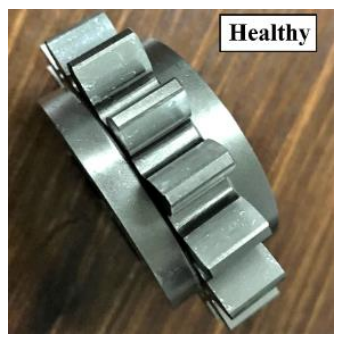

(a) Status of tooth surface

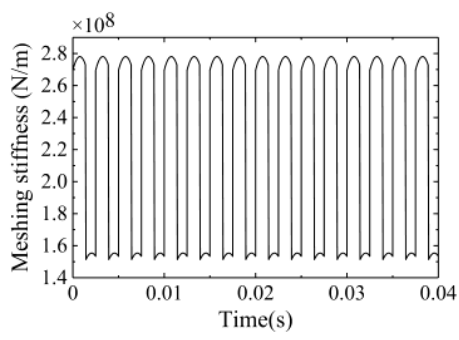

(b) Meshing stiffness curves
Figure 7 Status of tooth surface and meshing stiffness curves (healthy pinion)

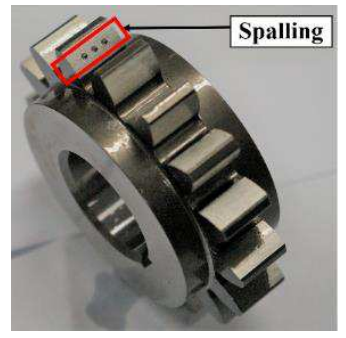

(a) Status of tooth surface

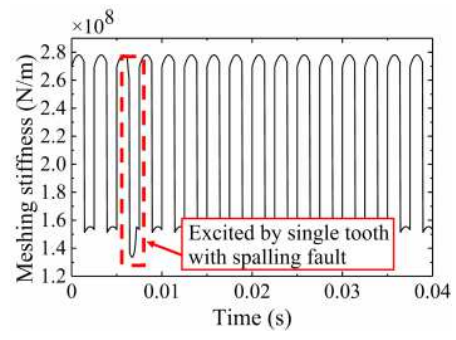

(b) Meshing stiffness curves
Figure 8 Status of tooth surface and meshing stiffness curves (single tooth with spalling fault)

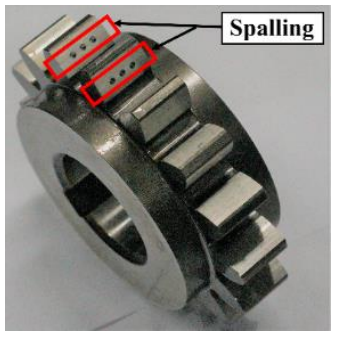

(a) Status of tooth surface

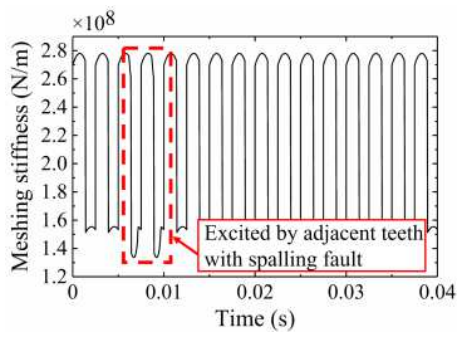

(b) Meshing stiffness curves
Figure 9 Status of tooth surface and meshing stiffness curves (adjacent teeth with spalling fault)

The simulated vibration signal and the experimental measurement signal under different number of spalling faulty tooth are displayed in Figures 10-12. The simulated results are close to the experimental results when the pinion is in a healthy state, as shown in Figure 10. There are no obvious impulse vibrations in the time domain, only tooth meshing frequency (TMF) and its harmonics are included in the frequency domain. In the frequency domain, the TMF is equal to the meshing frequency of gears, and the remaining harmonics are multiples of the TMF, as marked in Figure 10(b) and Figure 10(d).

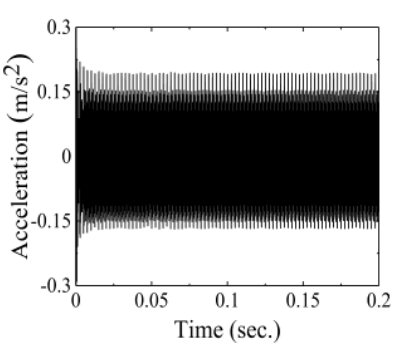

(a) Simulated signal

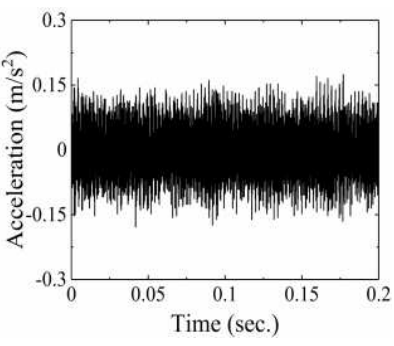

(c) Experimental signal

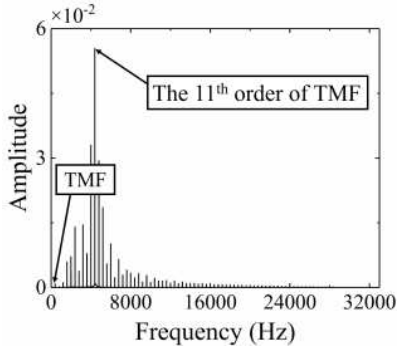

(b) Simulated frequency signal

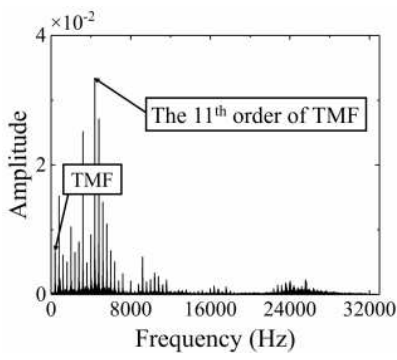

(d) Experimental frequency signal
Figure 10 The simulated and experimental signal in the meshing line direction (healthy pinion)

Compared with the healthy gear, the spalling fault can generate the periodic impulse vibration, and sidebands are spaced by the rotational frequency of the faulty gears in the frequency domain. These features are successfully captured by the simulated signal. Figure 11 shows the simulated and experimental signal results of the pinion with single tooth spalling fault. As shown in Figure 11, the periodic impulse vibration can be recognized in the time domain, and the period of the impulse vibration is $0.04 \mathrm{~s}$, which is equal to the rotating period of the pinion. The sideband appears in the frequency domain, and the spacing of the sideband is equal to the rotating frequency of the pinion. Figure 12 shows the simulated and experimental signal results of the pinion with adjacent double tooth spalling fault. Compared with the time domain in Figure 11(a), Figure 12(a) shows that the impulse vibration excited twice with an interval of $0.0025 \mathrm{~s}$ in one rotational period of the pinion. However, impulse vibration in the experimental signal is submerged by the background noise, as shown in Figure 11(c) and Figure 12(c). In the 
frequency domain, the maximum value of the sideband of Figure 12 is higher than that in Figure 11. The results of frequency domain analysis show that the fault feature in a case of double-teeth spalling are more obvious than that in case of single tooth spalling.

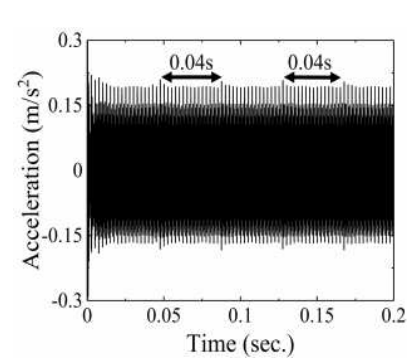

(a) Simulated signal

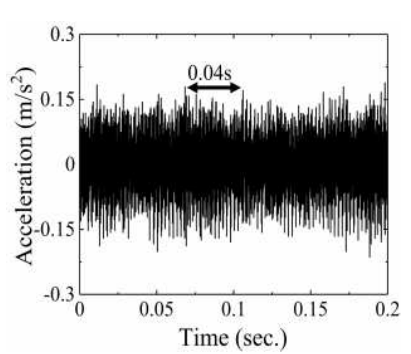

(c) Experimental signal

Figure 11 The simulated and experimental signal in the meshing line direction (single tooth with spalling fault)

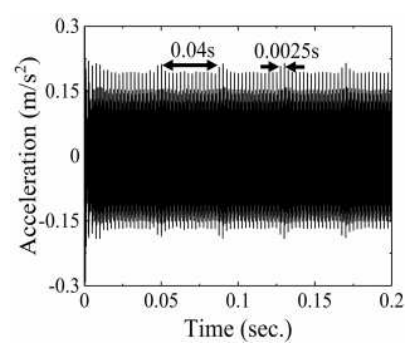

(a) Simulated signal

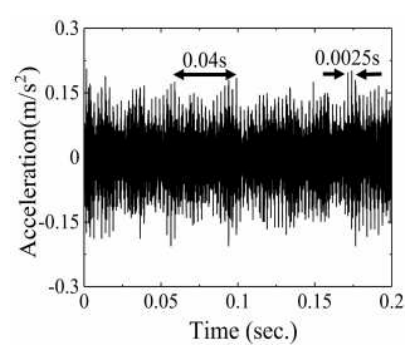

(c) Experimental signal

Figure 12 The simulated and experimental signal along the line of action (adjacent teeth spalling fault)

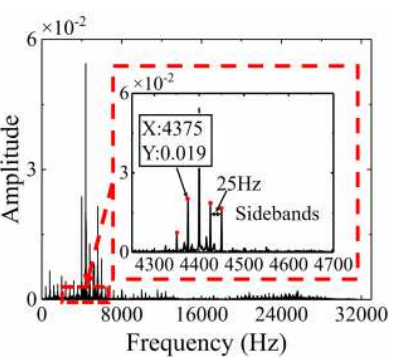

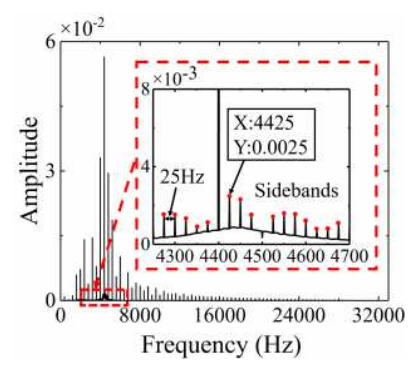

(b) Simulated frequency signal

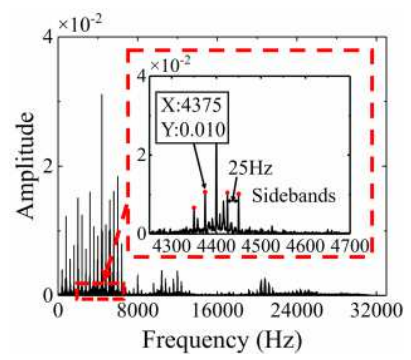

(d) Experimental frequency signal

\subsection{Effect of the relative position of the faulty teeth on dynamic characteristics of gears}

Based on the simulated and experimental parameters in Section 4.1, this paper we assume that the number of healthy teeth between two spalling faulty teeth is $H$. Three relative positions $(H=0, H=1$, and $H=2)$ of the two faulty teeth are considered. Figure 13(a), Figure 14(a) and Figure15(a) show the failure states of tooth surface spalling in three relative positions. Furthermore, when the two spalling fault teeth are meshed separately, the meshing stiffness decreases, and the interval time of the reductions is related to the number of healthy teeth between the two spalling fault teeth, as shown in Figure 14(b) and Figure15(b).

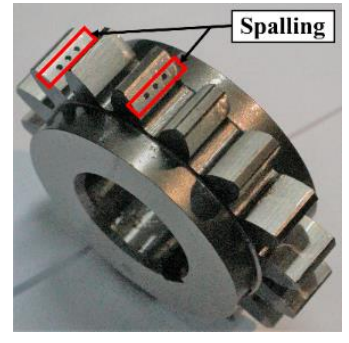

(a) Status of tooth surface

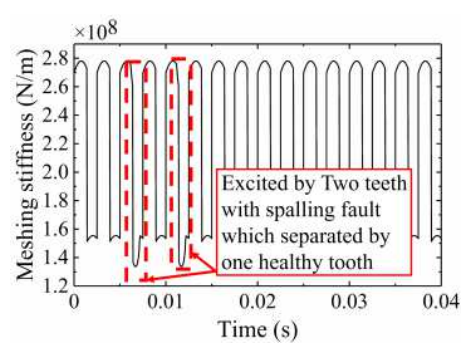

(b) Meshing stiffness curves
Figure 13 Status of tooth surface and meshing stiffness curves (two teeth with spalling fault which separated by one healthy tooth)

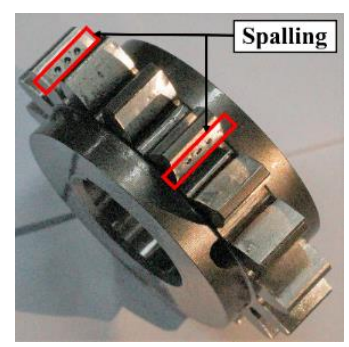

(a) Status of tooth surface

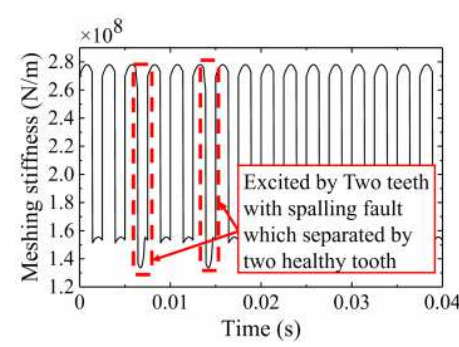

(b) Meshing stiffness curves
Figure 14 Status of tooth surface and meshing stiffness curves (two teeth with spalling fault which separated by two healthy teeth)

The simulated and experimental signals both in time and frequency domain at the three relative positions of the double faulty teeth are presented in Figure 12, Figure 15, and Figure 16. As shown in Figure 12(a), Figure 15(a), and Figure 16(a), impulse vibration excited twice in one rotational period of the pinion. Also, the distance between two impulse vibrations are $0.0025 \mathrm{~s}, 0.005 \mathrm{~s}$, and $0.0075 \mathrm{~s}$, which can be expressed by $(H+1) t_{m}$. However, the impulse vibration is not obvious in Figure 12(c), Figure 15(c), and 
Figure 16(c). One possible explanation is that these features are submerged by the background noise in the experimental signal.

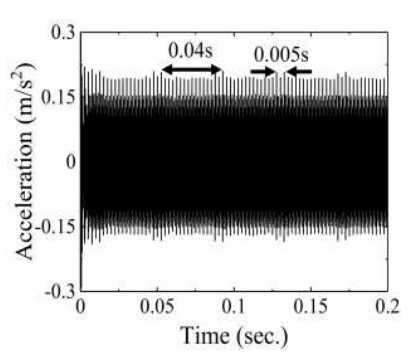

(a) Simulated signal

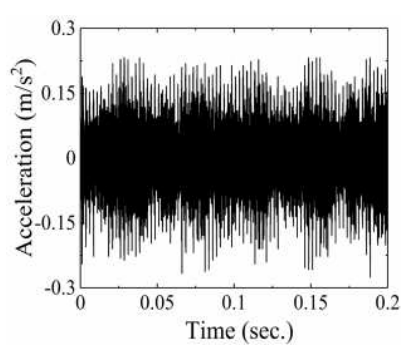

(c) Experimental signal

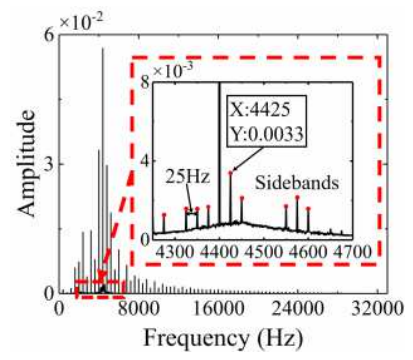

(b) Simulated frequency signal

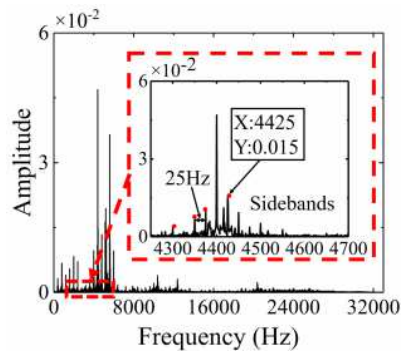

(d) Experimental frequency signal
Figure 15 The simulated and experimental signal in the meshing line direction (two teeth with spalling fault which separated by one healthy tooth)

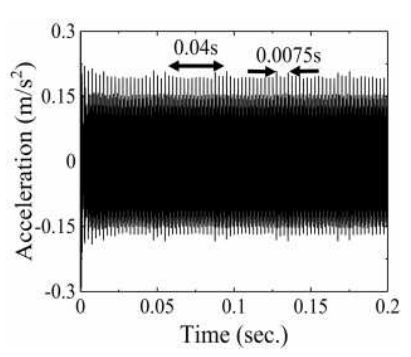

(a) Simulated signal

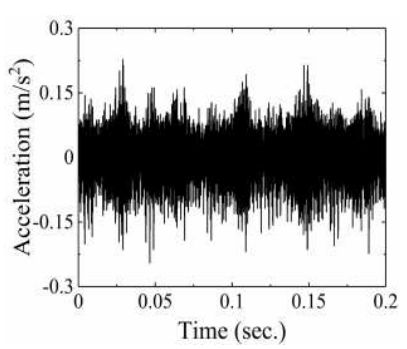

(c) Experimental signal

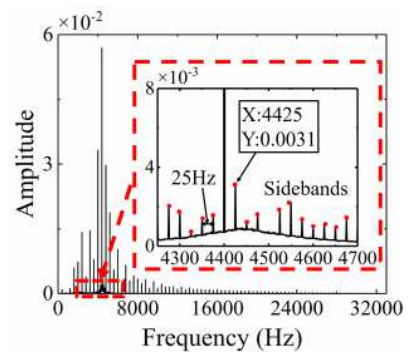

(b) Simulated frequency signal

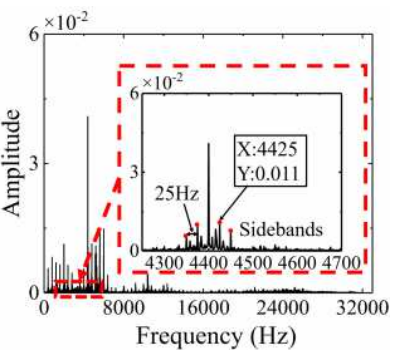

(d) Experimental frequency signal
Figure 16 The simulated and experimental signal in the meshing line direction (two teeth with spalling fault which separated by two healthy teeth)

Moreover, as presented in Figure 12(b), Figure 15(b), and Figure 16(b), the maximum amplitude of the sidebands at the three relative positions is $0.019,0.015$, and 0.011 , respectively. Among them, the amplitude of the sidebands at the adjacent positions is the highest, which is close to the simulated results. It can be concluded that when the two teeth with spalling fault are adjacent, the features of the spalling fault is the most obvious.

\subsection{Effect of rotational speed on dynamic characteristics of gears with double-teeth spalling fault}

Many statistical indicators based on time-domain signals can be well applied to the fault diagnosis of gear systems [50]. The pinion with adjacent spalling teeth is taken as an example for exploring the influence of the rotational speed on the gear dynamic characteristics by using three statistical indicators in this paper. Three types of rotational speed ( $n=1500 \mathrm{r} / \mathrm{min}, n=3000 \mathrm{r} / \mathrm{min}$, and $n=4500 \mathrm{r} / \mathrm{min}$ ) of the pinion are considered.

RMS is used for measuring the overall vibration level, which is expressed as [51]:

$$
R M S=\sqrt{\frac{1}{N} \sum_{n=1}^{N}\left(x(n)-\frac{1}{n} \sum_{n=1}^{N} x(n)\right)^{2}},
$$

in which $N$ is the number of data points in the signal, and $x(n)$ is the amplitude of the $n^{\text {th }}$ point.

Kurtosis [52] is a parameter sensitive to the shape of signal, and can detect the impulse signal caused by local fault well, which is defined by Eq. (16).

$$
\text { Kurtosis }=\frac{(1 / N) \sum_{n=1}^{N}(x(n)-\bar{x})^{4}}{\left[(1 / N) \sum_{n=1}^{N}(x(n)-\bar{x})^{2}\right]^{2}}
$$

Third statistical moment parameter $S_{a}$ [53] is similar to the kurtosis in impulse recognition, but it is more sensitive to the impulse, which can be calculated by Eq. (17).

$$
S_{a}=\frac{(1 / N) \sum_{n=1}^{N}|x(n)-\bar{x}|^{3}}{\left[(1 / N) \sum_{n=1}^{N}|x(n)-\bar{x}|\right]^{3}}
$$

Table 2 shows the statistical characteristics under different rotational speed of the pinion. With the increase of the rotational speed, the values of RMS both in simulation and experimental results increase, and the values of Kurtosis and $S_{a}$ decrease in the simulation results. Contrary to the simulation results, the experimental results demonstrate that the values of Kurtosis and $S_{a}$ increase when the rotational speed is between $3000 \mathrm{rpm}$ and 4500 rpm. This inconsistency may be caused by the vibration of 
the whole experimental device due to the increase of the rotational speed. The results of the experimental (EXPM) are consistent with the simulated (SIM) in general.

Table 2 The effect of rotation speed on statistical indicators

\begin{tabular}{|c|c|c|c|c|c|c|}
\hline \multirow{2}{*}{$\begin{array}{l}\text { RMP } \\
(\mathrm{r} / \mathrm{min})\end{array}$} & \multicolumn{2}{|r|}{ RMS } & \multicolumn{2}{|c|}{ Kurtosis } & \multicolumn{2}{|r|}{$S_{a}$} \\
\hline & SIM & EXPM & SIM & EXPM & SIM & EXPM \\
\hline 1500 & 1.225 & 5.573 & 10.376 & 3.189 & 17.098 & 3.328 \\
\hline 3000 & 1.857 & 9.132 & 6.452 & 2.927 & 5.336 & 3.065 \\
\hline 4500 & 15.912 & 17.877 & 2.410 & 3.107 & 2.565 & 3.230 \\
\hline
\end{tabular}

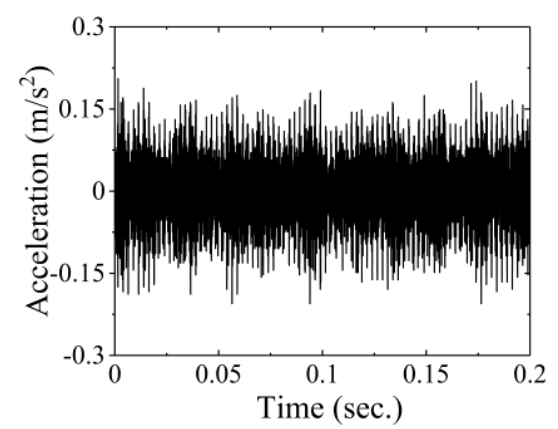

(a) $1500 \mathrm{r} / \mathrm{min}$

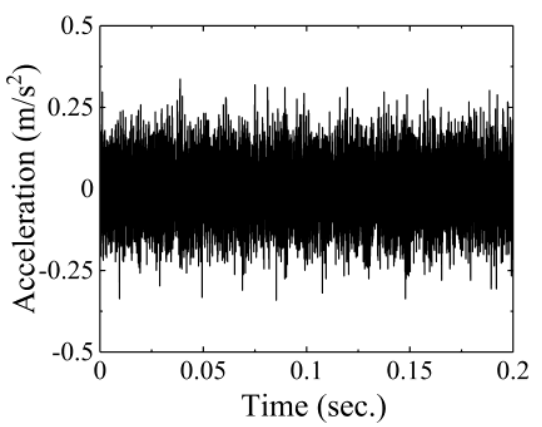

(b) $3000 \mathrm{r} / \mathrm{min}$

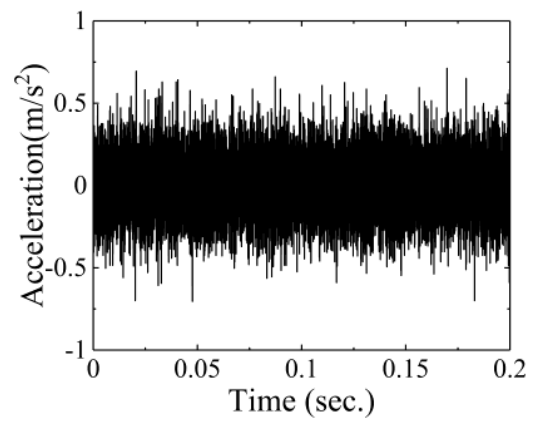

(c) $4500 \mathrm{r} / \mathrm{min}$

Figure 17 The experimental time-domain signal in the direction of meshing line under three pinion rotational speed (pinion with adjacent spalling teeth)

Furthermore, it can be found that the values of Kurtosis and $S_{a}$ reach the maximum at a rotational speed of $n=1500$ r/min. The results indicate that the features of the double-teeth spalling fault is more obvious at low rotational speed. With the increase of the rotational speed of pinion, the impulse vibration is gradually submerged by the background noise. This conclusion is also confirmed by the experimental results, as shown in Figure 17.

\section{Feature extraction method of double - teeth spalling fault}

To solve the problem that the features of double-teeth spalling fault is submerged by the complex background noise in the experimental signal, a feature extraction method based on the VMD and the FK is proposed. The experimental signal is denoised by using the VMD method, which can ensure the effectiveness of the FK in fault feature extraction. The reconstructed signal is filtered by an optimal band-pass filter based on the FK.

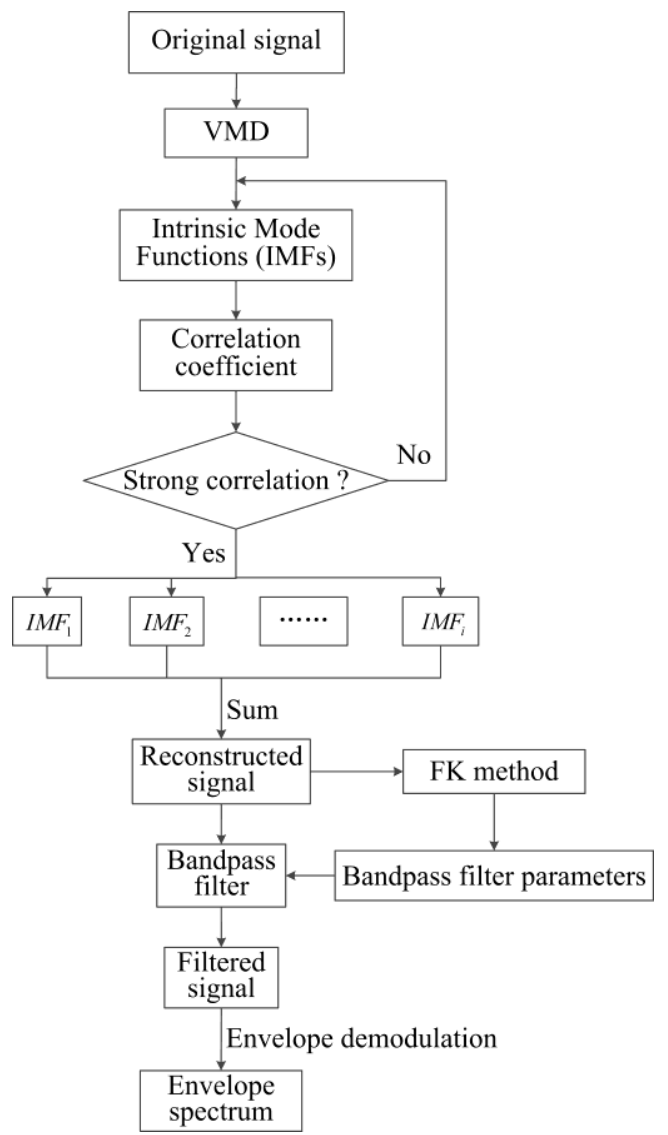

Figure 18 The procedure of the feature extraction method based on the VMD and the FK

Figure 18 outlines the procedure of the proposed method, and the major steps are as follows:

Step 1: the original signal with the double-teeth spalling fault collected from experiments is decomposed into a set 
of IMFs by using the VMD method.

Step 2: the correlation coefficient between each IMFs and the original signal is calculated, and the IMFs (i.e., the IMFs with a correlation coefficient greater than 0.6) which conforms to the strong correlation criterion are summed as the reconstructed signal.

Step 3: based on the FK, the frequency and bandwidth of the signal which contains the most information of the double-teeth spalling fault feature are adaptively selected as the parameters of the band-pass filter.

Step 4: the filtered signal is demodulated by the envelope analysis.

Step 5: the time domain and the envelope spectrum of the filtered signal are utilized to analyze the features of the double-teeth spalling fault.

According to the conclusions given in Section 4, the features of the double-teeth spalling fault are extracted by using the method described in this Section. The experimental signal when the two spalling faulty teeth are adjacent and the rotational speed $n=1500 \mathrm{r} / \mathrm{min}$ is taken as the object, as shown in Figure 12(c) and Figure 12 (d).
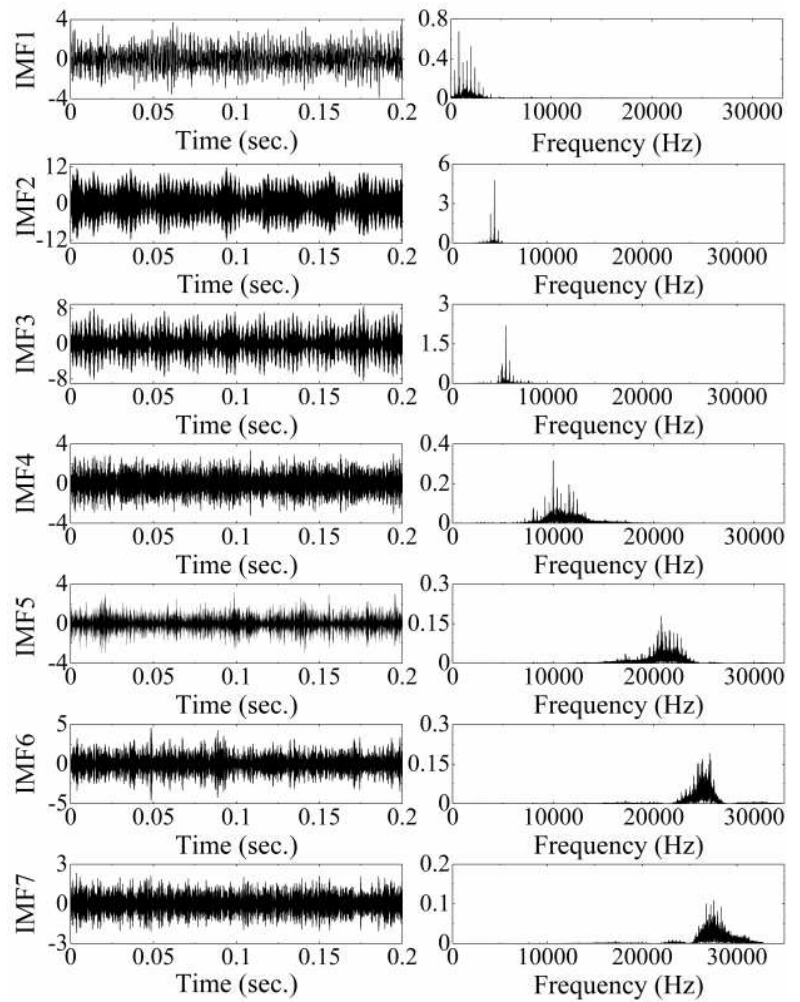

Figure 19 Results of decomposition based on the VMD for the experimental signal

Compared with the simulated signal, the features of double-teeth spalling fault can not be clearly observed in the experimental signal because of the complex background noise. To address this problem, the VMD is used to decompose the original signal with double-teeth spalling fault which collected in the experiment into a set of IMFs. According to the central frequency approach principle, the value of decomposition level is set to 7 , the value of penalty factor is set to 2000, the value of Lagrange multiplier is set to 0 , and the results of the decomposition are shown in Figure 19.

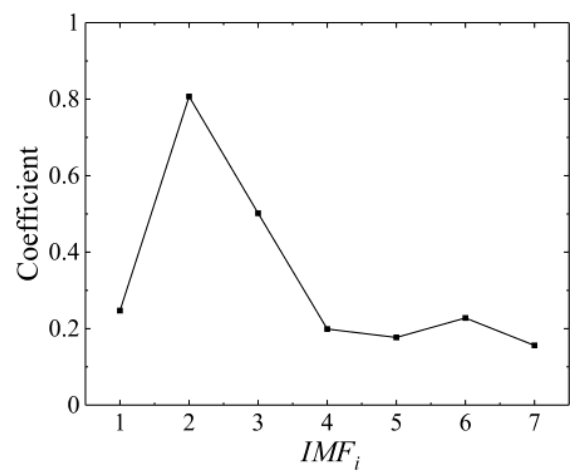

Figure 20 The correlation coefficient between each IMF and the original experimental signal

The correlation coefficient between each IMF and the original signal is calculated. As presented in Figure 20, only the correlation coefficient value of $\mathrm{IMF}_{2}$ is greater than 0.6. According to the correlation coefficient algorithm, $\mathrm{IMF}_{2}$ is selected as the reconstructed signal.

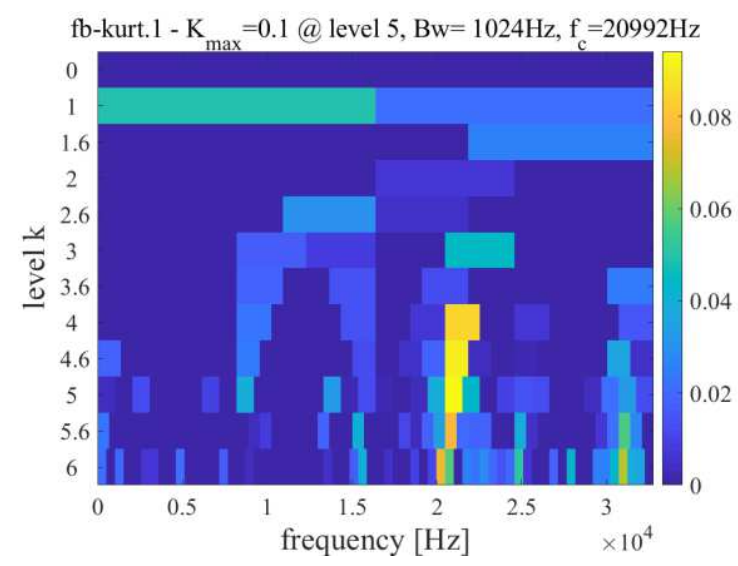

Figure 21 The Kurtogram of the reconstructed signal

The frequency and bandwidth of the IMFs which contain the most information of the double-teeth spalling fault are adaptively selected as the reconstructed signal by using the FK method. As shown in Figure 21, the frequency value is $20992 \mathrm{~Hz}$, and the bandwidth is 1024 Hz. The frequency and bandwidth of IMFs are used as 
band-pass filter parameters to filter the reconstructed signal. Subsequently, the filtered signal is demodulated by the envelope analysis, and the results of the reconstructed signal and the envelope spectrum are shown in Figure 22.

Compared with Figure 12(c), the periodic impulse vibration which excited by the spalling fault can be clearly observed in Figure 22(a). The period of the impulse vibration is $0.04 \mathrm{~s}$, which is equal to the rotating period of the pinion. Also, in a rotation period of the pinion, the impulse vibration excited twice, and the interval time of the two impulse vibration is close to the single tooth meshing period. This is consistent with the previous simulated results mentioned in Section 4.

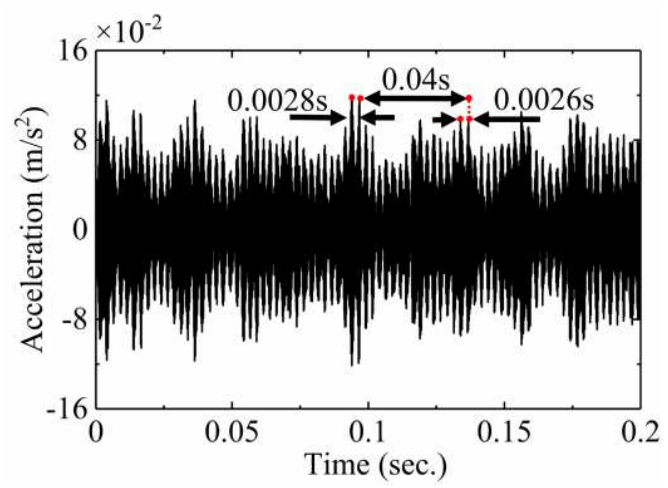

(a)The time-domain filtered signal

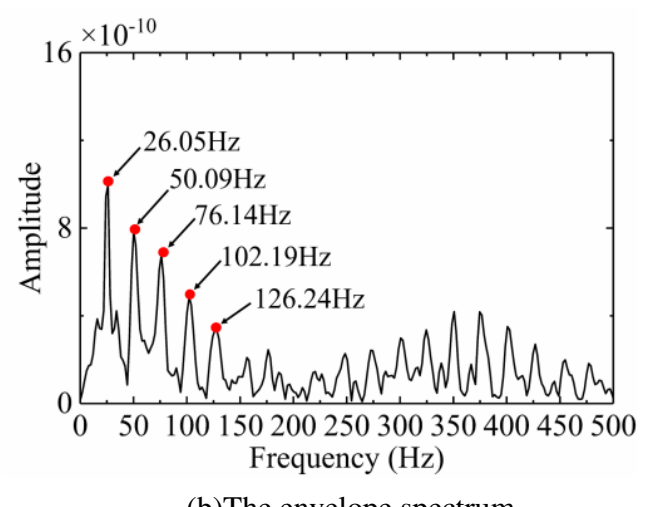

(b)The envelope spectrum

Figure 22 The results of the proposed method based on VMD and FK for the gear system experimental signal with double-teeth spalling fault

The performance of the proposed method is compared with the original FK method and the method combined with the EMD and the FK. Figure 22(b), Figure 23, and Figure 24 show the envelop spectrum of the proposed method, the original FK method, and the method combined with the EMD and the FK. For clarity, we set the visible abscissa range of the spectrum to $0-500 \mathrm{~Hz}$. As can be observed in Figure 22(b), five spectrum lines are located at the harmonics of the pinion rotating frequency, which are
26.05 Hz, 50.09 Hz, 76.14 Hz, 102.19 Hz, and 126.24 Hz. The results of frequency domain analysis strongly demonstrate that the pinion is a faulty gear. Compared with the phenomenon in Figure 22(b), there is one fault frequency harmonic in Figure 23, and two fault frequency harmonics in Figure 24. Besides, considerable background noises exist in Figure 23 and 24. Therefore, it can conclude that the proposed method outperformed the original FK and the method combined with the EMD and the FK in the feature extraction of gear fault.

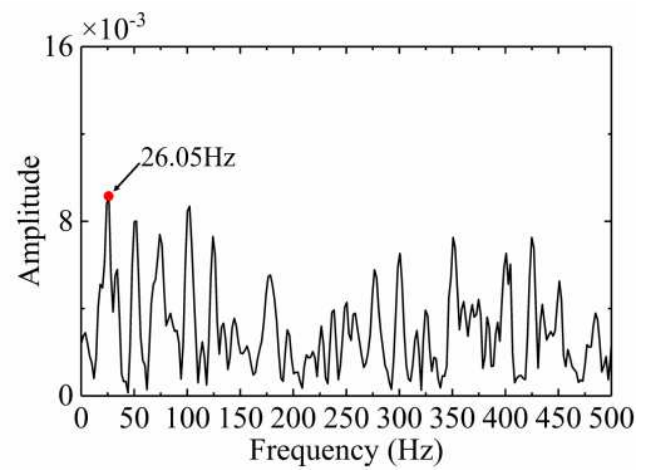

Figure 23 The envelop spectrum of the original FK method for the gear system experimental signal with double-teeth spalling fault

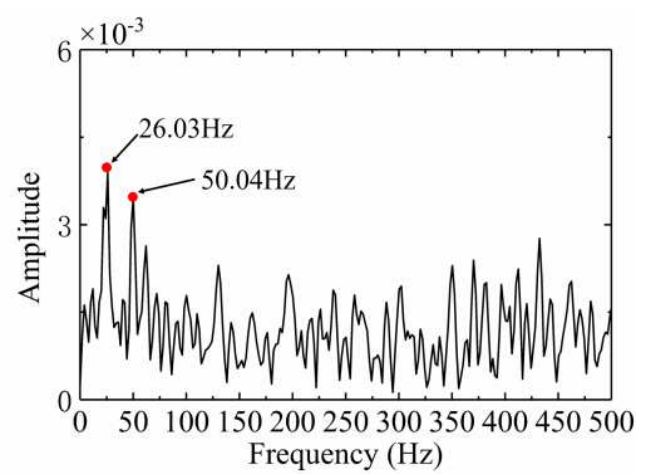

Figure 24 The envelop spectrum of the method combined with the EMD and the FK for the gear system experimental signal with double-teeth spalling fault

\section{Conclusions}

In this paper, a dynamic model of a gear system with double-teeth spalling fault is presented with considering the influence of TVMS, transmission error, and bearing support stiffness. The effectiveness of the proposed dynamic model is validated by analyzing experimental results. The effect of the number of spalling teeth, the relative position of the two faulty teeth, and the rotational speed on the dynamic characteristics are investigated. Both 
of the experimental test and dynamic simulation results have shown that the double-teeth spalling fault will generate impulse vibration twice in one rotating period of the fault gear, and the interval of the two impulse is related to the number of healthy teeth, which can be expressed as $(H+1) t_{m}$. Moreover, the features of double-teeth spalling fault are significant when the two spalling teeth are adjacent and the pinion runs at a low rotational speed.

A feature extraction method which combines the VMD and the FK is proposed. The raw signal obtains from experiments is decomposed by using the VMD. The IMFs that reserve a strong correlation with the original signal are summed as a reconstructed signal according to the value of the coefficient correlation. The reconstructed signal is filtered by the optimal filter based on the FK. The results confirm that, (1) the VMD has a good denoising ability and can provide the FK with a well denoised signal; (2) By compared with the original FK method and the method based on the EMD and the FK, it can be found that the proposed VMD-FK method is more effective in extracting the features of the double-teeth spalling fault in gear fault diagnosis than other methods.

\section{Declaration}

\section{Acknowledgements}

The authors sincerely thanks to Shuanghuan Driveline Co., Ltd. for providing the necessary facilities and machinery to build the prototype of the gear system.

\section{Funding}

Supported by National Natural Science Foundation of China (Grant No. 51475425).

\section{Availability of data and materials}

The datasets supporting the conclusions of this article are included within the article.

\section{Authors' contributions}

The author' contributions are as follows: BP was in charge of the whole study; LS, JW and YX wrote the manuscript; QZ and CL assisted with sampling and laboratory analyses. All authors read and approved the final manuscript.

\section{Competing interests}

The authors declare no competing financial interests.

\section{Consent for publication}

Not applicable

Ethics approval and consent to participate

Not applicable

\section{References}

[1] A Kumar, C P Gandhi, Y Zhou, et al. Latest developments in gear defect diagnosis and prognosis: A review. Measurement, 2020, 158: 107735.

[2] T Davidson, P Ku. The effect of lubricants on gear tooth surface fatigue. ASLE Transactions, 1958, 1(1): 40-50.

[3] Z Chen, W Zhai, K Wang. Locomotive dynamic performance under traction/braking conditions considering effect of gear transmissions. Vehicle System Dynamics, 2018, 56(7): 1097-1117.

[4] K Feng, P Borghesani, W A Smith, et al. Vibration-based updating of wear prediction for spur gears. Wear, 2019, 426: 1410-1415.

[5] M Weibring, L Gondecki, P Tenberge. Simulation of fatigue failure on tooth flanks in consideration of pitting initiation and growth. Tribology International, 2019, 131: 299-307.

[6] Z Zhang, S Li, J Wang, et al. Enhanced sparse filtering with strong noise adaptability and its application on rotating machinery fault diagnosis. Neurocomputing, 2020. (https://doi.org/10.1016/j.neucom.2020.02.042)

[7] Y Ding, N F Rieger. Spalling formation mechanism for gears. Wear, 2003, 254(12): 1307-1317.

[8] X Liang, M J Zuo, Z Feng. Dynamic modeling of gearbox faults: A review. Mechanical Systems and Signal Processing, 2018, 98: 852-876.

[9] H Ma, Z Li, M Feng, et al. Time-varying mesh stiffness calculation of spur gears with spalling defect. Engineering Failure Analysis, 2016, 66: 166-176.

[10] A Saxena, A Parey, M Chouksey. Time varying mesh stiffness calculation of spur gear pair considering sliding friction and spalling defects. Engineering Failure Analysis, 2016, 70: 200-211.

[11] X Liang, $\mathrm{H}$ Zhang, L Liu, et al. The influence of tooth pitting on the mesh stiffness of a pair of external spur gears. Mechanism and Machine Theory, 2016, 106: 1-15.

[12] Y Luo, N Baddour, G Han, et al. Evaluation of the time-varying mesh stiffness for gears with tooth spalls with curved-bottom features. Engineering Failure Analysis, 2018, 92: 430-442.

[13] T Chen, Y Wang, Z Chen. A novel distribution model of multiple teeth pits for evaluating time-varying mesh stiffness of external spur gears. Mechanical Systems and Signal Processing, 2019, 129: 479-501.

[14] Y Luo, $N$ Baddour, $M$ Liang. Dynamical modeling and experimental validation for tooth pitting and spalling in spur gears. Mechanical Systems and Signal Processing, 2019, 119: 155-181.

[15] P J Blau. Friction, lubrication, and wear technology. ASM International, 1992: 175-235.

[16] C K Tan, P Irving, D Mba. A comparative experimental study on the diagnostic and prognostic capabilities of acoustics emission, vibration and spectrometric oil analysis for spur gears. Mechanical Systems and Signal Processing, 2007, 21(1): 208-233.

[17] X-H Liang, Z-L Liu, J Pan, et al. Spur gear tooth pitting propagation assessment using model-based analysis. Chinese Journal of Mechanical Engineering, 2017, 30(6): 1369-1382.

[18] R Dwyer. Detection of non-Gaussian signals by frequency domain 
kurtosis estimation. ICASSP'83 IEEE International Conference on Acoustics, Speech, and Signal Processing, Boston, 1983: 607-610.

[19] J Antoni. The spectral kurtosis: a useful tool for characterising non-stationary signals. Mechanical Systems and Signal Processing, 2006, 20(2): 282-307.

[20] J Antoni, R Randall. The spectral kurtosis: application to the vibratory surveillance and diagnostics of rotating machines. Mechanical Systems and Signal Processing, 2006, 20(2): 308-331.

[21] J Antoni. Fast computation of the kurtogram for the detection of transient faults. Mechanical Systems and Signal Processing, 2007, 21(1): 108-124.

[22] H Wang, J Chen, G Dong. Fault diagnosis of rolling bearing's early weak fault based on minimum entropy de-convolution and fast Kurtogram algorithm. Proceedings of the Institution of Mechanical Engineers, Part C: Journal of Mechanical Engineering Science, 2015, 229(16): 2890-2907.

[23] L Wang, Z Liu, Q Miao, et al. Time-frequency analysis based on ensemble local mean decomposition and fast kurtogram for rotating machinery fault diagnosis. Mechanical Systems and Signal Processing, 2018, 103: 60-75.

[24] B Chen, Z Zhang, Y Zi, et al. Detecting of transient vibration signatures using an improved fast spatial-spectral ensemble kurtosis kurtogram and its applications to mechanical signature analysis of short duration data from rotating machinery. Mechanical Systems and Signal Processing, 2013, 40(1): 1-37.

[25] S Prabhakar, A Sekhar, A Mohanty. Detection and monitoring of cracks in a rotor-bearing system using wavelet transforms. Mechanical Systems and Signal Processing, 2001, 15: 447-450.

[26] P Liang, C Deng, J Wu, et al. Compound Fault Diagnosis of Gearboxes via Multi-label Convolutional Neural Network and Wavelet Transform. Computers in Industry, 2019, 113: 103132.

[27] N E Huang, Z Shen, S R Long, et al. The empirical mode decomposition and the Hilbert spectrum for nonlinear and non-stationary time series analysis. Proceedings of the Royal Society of London Series A: mathematical, physical and engineering sciences, 1998, 454(1971): 903-995.

[28] A Rai, S Upadhyay. Bearing performance degradation assessment based on a combination of empirical mode decomposition and k-medoids clustering. Mechanical Systems and Signal Processing, 2017, 93: 16-29.

[29] T Guo, Z Deng. An improved EMD method based on the multi-objective optimization and its application to fault feature extraction of rolling bearing. Applied Acoustics, 2017, 127: 46-62.

[30] S Osman, W Wang. A morphological Hilbert-Huang transform technique for bearing fault detection. IEEE Transactions on Instrumentation and Measurement, 2016, 65(11): 2646-2656.

[31] L Mingliang, W Keqi, S Laijun, et al. Applying empirical mode decomposition (EMD) and entropy to diagnose circuit breaker faults. Optik, 2015, 126(20): 2338-2342.

[32] J B Ali, N Fnaiech, L Saidi, et al. Application of empirical mode decomposition and artificial neural network for automatic bearing fault diagnosis based on vibration signals. Applied Acoustics, 2015, 89: 16-27.

[33] Z Wu, N E Huang. Ensemble empirical mode decomposition: a noise-assisted data analysis method. Advances in adaptive data analysis, 2009, 1(01): 1-41.

[34] K Yu, T R Lin, J W Tan. A bearing fault diagnosis technique based on singular values of EEMD spatial condition matrix and Gath-Geva clustering. Applied Acoustics, 2017, 121: 33-45.

[35] J S Smith. The local mean decomposition and its application to
EEG perception data. Journal of the Royal Society Interface, 2005, 2(5): 443-454.

[36] Z Liu, Z He, W Guo, et al. A hybrid fault diagnosis method based on second generation wavelet de-noising and local mean decomposition for rotating machinery. ISA Transactions, 2016, 61: 211-220.

[37] K Dragomiretskiy, D Zosso. Variational mode decomposition. IEEE Transactions on Signal Processing, 2013, 62(3): 531-544.

[38] Y Wang, F Liu, Z Jiang, et al. Complex variational mode decomposition for signal processing applications. Mechanical Systems and Signal Processing, 2017, 86: 75-85.

[39] Y Wang, R Markert. Filter bank property of variational mode decomposition and its applications. Signal Processing, 2016, 120: 509-521.

[40] M Zhang, Z Jiang, K Feng. Research on variational mode decomposition in rolling bearings fault diagnosis of the multistage centrifugal pump. Mechanical Systems and Signal Processing, 2017, 93: 460-493.

[41] X An, F Zhang. Pedestal looseness fault diagnosis in a rotating machine based on variational mode decomposition. Proceedings of the Institution of Mechanical Engineers, Part C: Journal of Mechanical Engineering Science, 2017, 231(13): 2493-2502.

[42] Y Li, G Li, Y Wei, et al. Health condition identification of planetary gearboxes based on variational mode decomposition and generalized composite multi-scale symbolic dynamic entropy. ISA Transactions, 2018, 81: 329-341.

[43] Y Li, G Cheng, C Liu, et al. Study on planetary gear fault diagnosis based on variational mode decomposition and deep neural networks. Measurement, 2018, 130: 94-104.

[44] F Li, R Li, L Tian, et al. Data-driven time-frequency analysis method based on variational mode decomposition and its application to gear fault diagnosis in variable working conditions. Mechanical Systems and Signal Processing, 2019, 116: 462-479.

[45] J Li, H Wang, J Zhang, et al. Impact fault detection of gearbox based on variational mode decomposition and coupled underdamped stochastic resonance. ISA Transactions, 2019, 95: 320-329.

[46] R Li, J Wang. Gear system dynamics. Beijing: Science Press, 1997.(in Chinese)

[47] F Chaari, T Fakhfakh, M Haddar. Analytical modelling of spur gear tooth crack and influence on gearmesh stiffness. European Journal of Mechanics-A/Solids, 2009, 28(3): 461-468.

[48] W Bartelmus. Mathematical modelling and computer simulations as an aid to gearbox diagnostics. Mechanical Systems and Signal Processing, 2001, 15(5): 855-871.

[49] H T Chen, X L Wu, D T Qin, et al. Effects of gear manufacturing error on the dynamic characteristics of planetary gear transmission system of wind turbine. Applied Mechanics and Materials, 2011: 518-522.

[50] V Sharma, A Parey. A review of gear fault diagnosis using various condition indicators. Procedia Engineering, 2016, 144: 253-263.

[51] B D Forrester. Advanced vibration analysis techniques for fault detection and diagnosis in geared transmission systems. Swinburne University of Technology Melbourne, VIC, Australia, 1996.

[52] J Dron, F Bolaers. Improvement of the sensitivity of the scalar indicators (crest factor, kurtosis) using a de-noising method by spectral subtraction: application to the detection of defects in ball bearings. Journal of Sound and Vibration, 2004, 270(1-2): 61-73.

[53] B Tao, L Zhu, H Ding, et al. An alternative time-domain index for condition monitoring of rolling element bearings-A comparison 
study. Reliability Engineering \& System Safety, 2007, 92(5): 660-670.

\section{Biographical notes}

Luo-Jie Shi, born in 1994, is currently a master candidate at College of Mechanical Engineering, Zhejiang University of Technology, China. His main research interests include machinery fault diagnostics, and reliability analysis.

E-mail: luojieshi@zjut.edu.cn

Juan Wen, born in 1990, is currently an assistant professor at College of Mechanical Engineering, Zhejiang University of Technology, China. Her main research interests include machinery condition monitoring, intelligent fault diagnosis, and remaining useful life prediction.

E-mail: juanwen@zjut.edu.cn

Bai-Song Pan, born in 1968, is currently an professor at College of Mechanical Engineering, Zhejiang University of Technology, China. His main research interests include reliability design, and machinery fault diagnostics.

E-mail: panbsz@zjut.edu.cn
Yong-Yong Xiang, born in 1991, is currently a $\mathrm{PhD}$ candidate at College of Mechanical Engineering, Zhejiang University of Technology, China. His main research interests include uncertainty analysis, and model validation and calculation.

E-mail: xiangyyong@126.com

Qi Zhang, born in 1984, is currently a senior engineer at Zhejiang Shuanghuan Driveline Co., Ltd., Zhejiang Province, China. His main research interests include mechanical system design \& NVH testing.

E-mail: zhangqi@gearsnet.com

Cong-Kai Lin, born in 1995, is currently a master candidate at College of Mechanical Engineering, Zhejiang University of Technology, China. His main research interests include reliability analysis method application and algorithm reaearch.

E-mail: lincongkai7980@163.com

\section{Appendix}

\section{Not applicable}


Figures
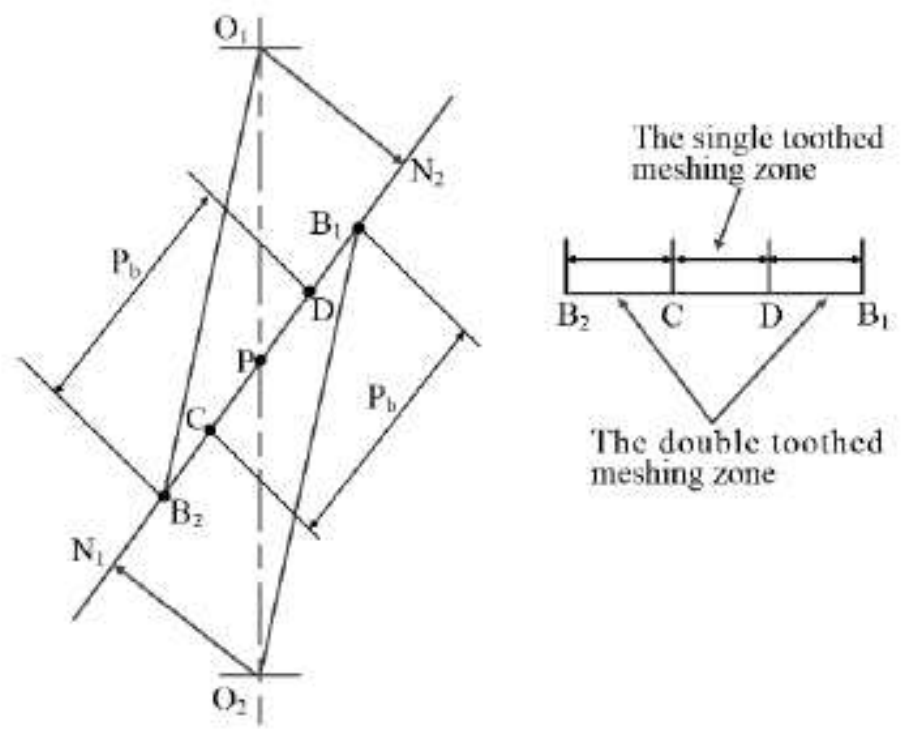

Figure 1

Schematic diagram of gear meshing

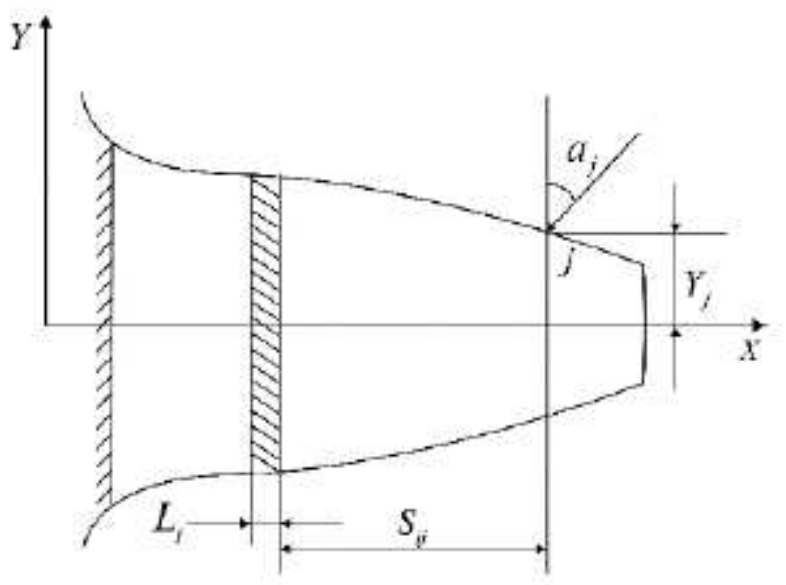

Figure 2

Schematic diagram of a gear tooth cantilever beam 

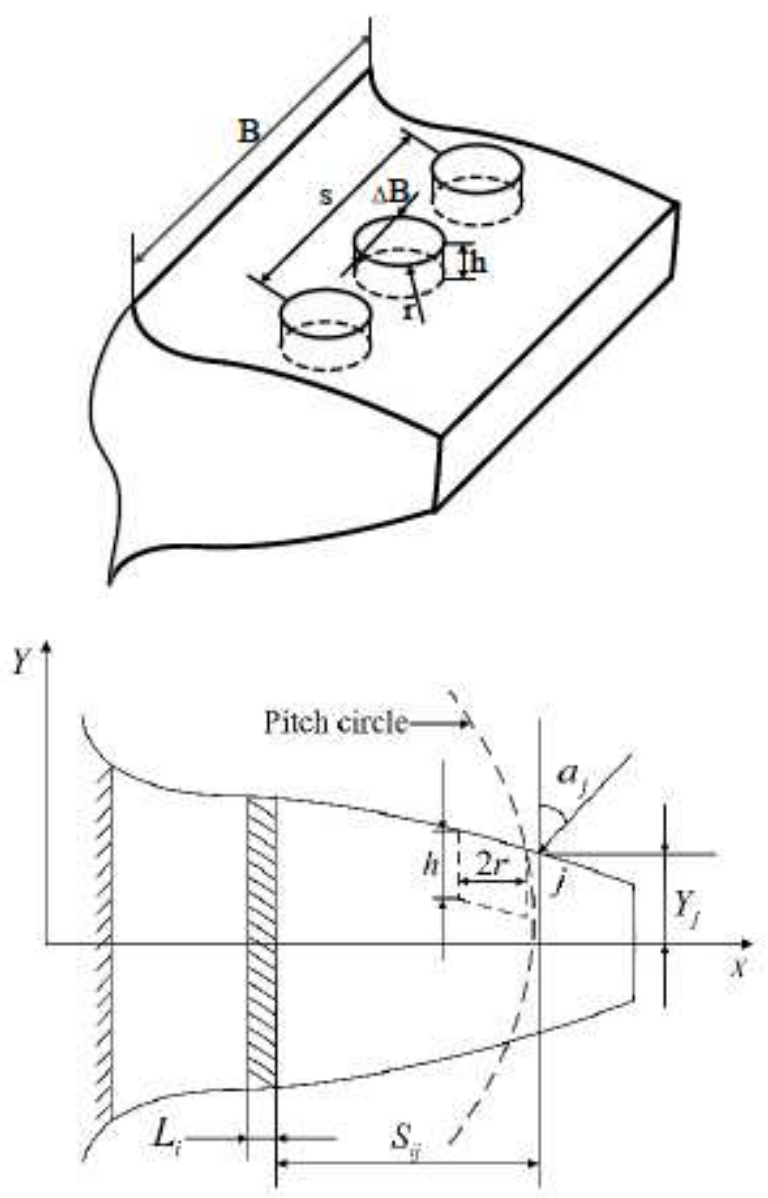

Figure 3

Geometric model of the spalling tooth

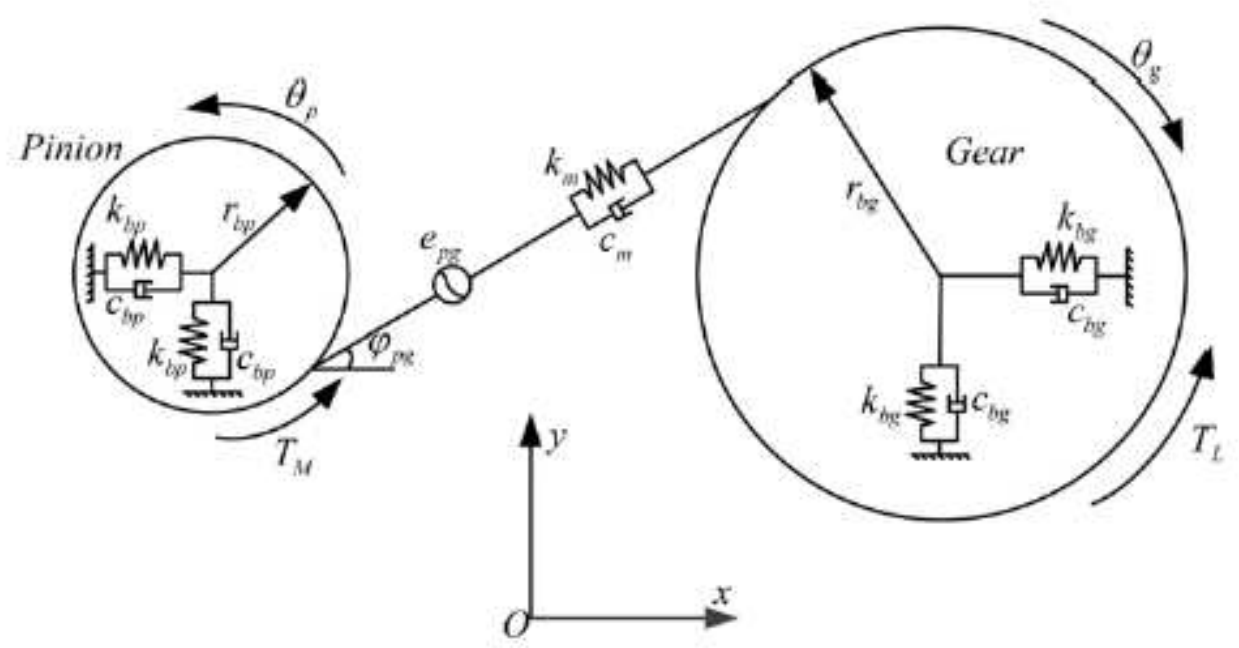

Figure 4

Dynamic model of the 6-DOF gear system 


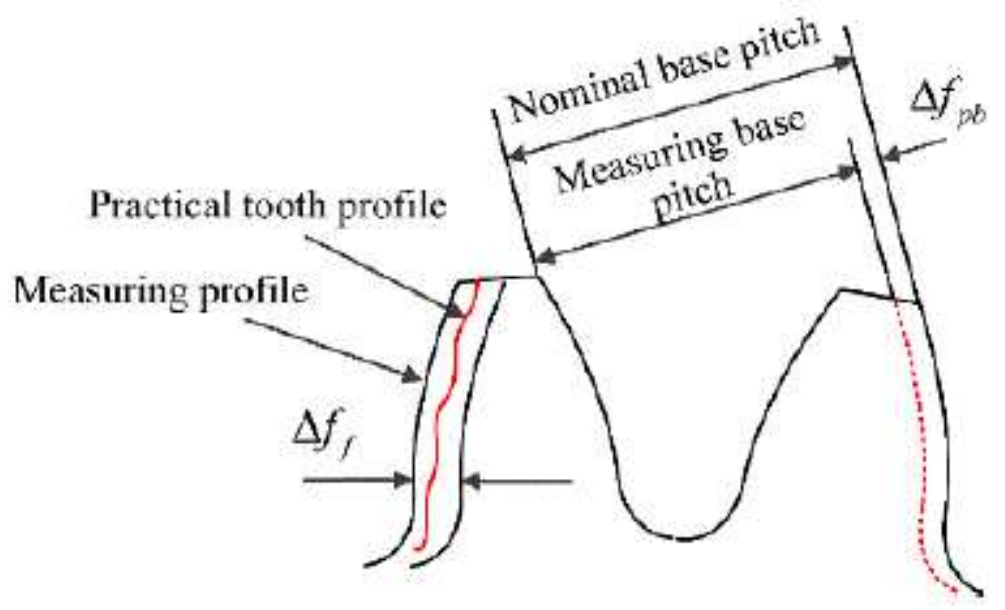

Figure 5

Tooth error and base pitch error[49]

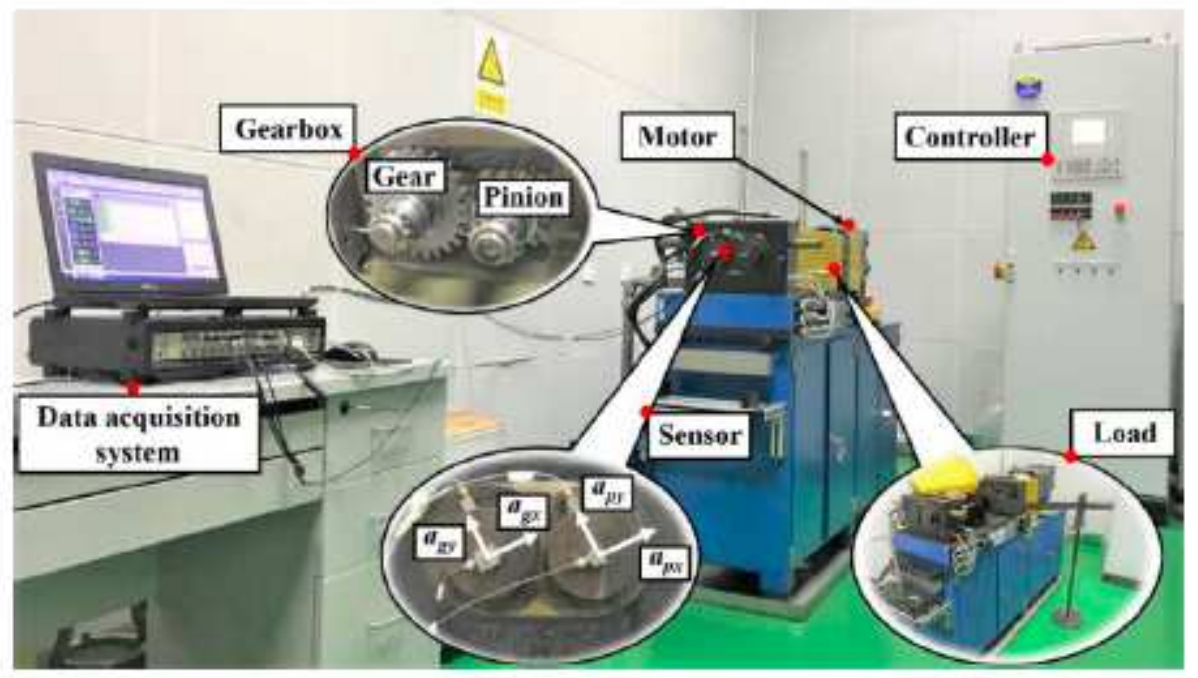

Figure 6

The FZG test-rig 


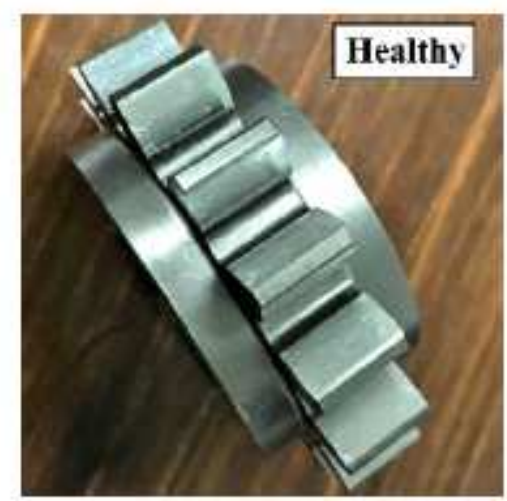

(a) Status of tooth surface

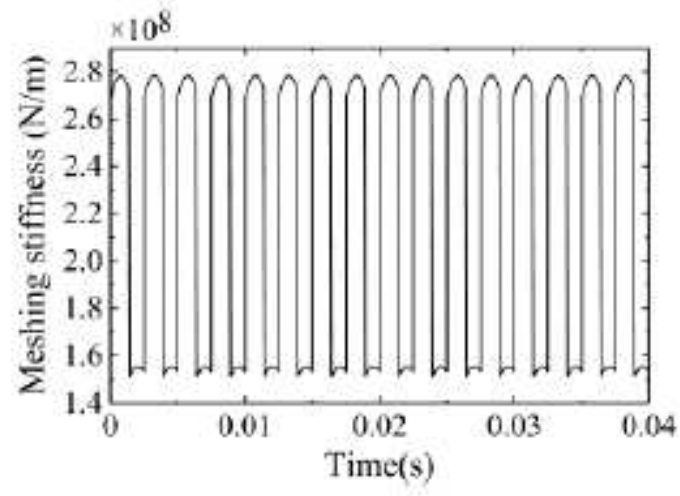

(b) Meshing stiffness curves

\section{Figure 7}

Status of tooth surface and mesh ing stiffness curves (healthy pinion)

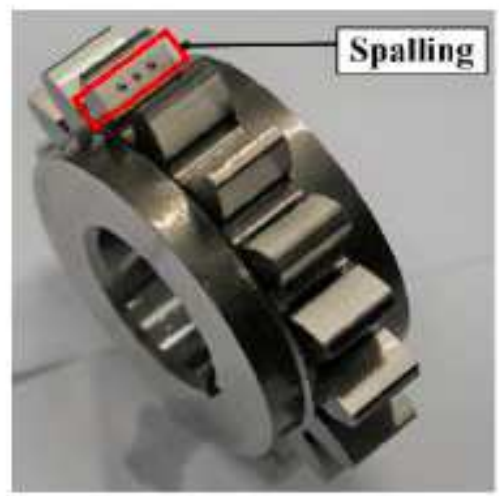

(a) Status of tooth surface

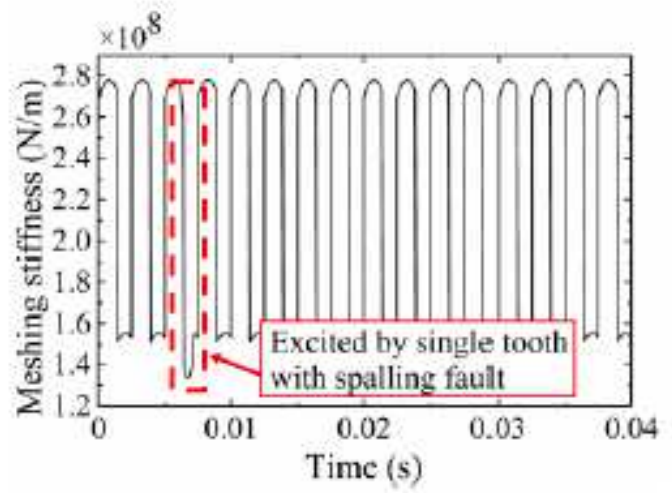

(b) Meshing stiffness curves

\section{Figure 8}

Status of tooth surface and mesh ing stiffness curves (single tooth with spalling fault)

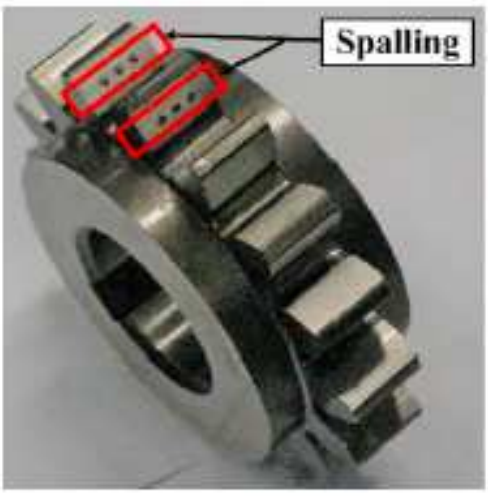

(a) Status of tooth surface

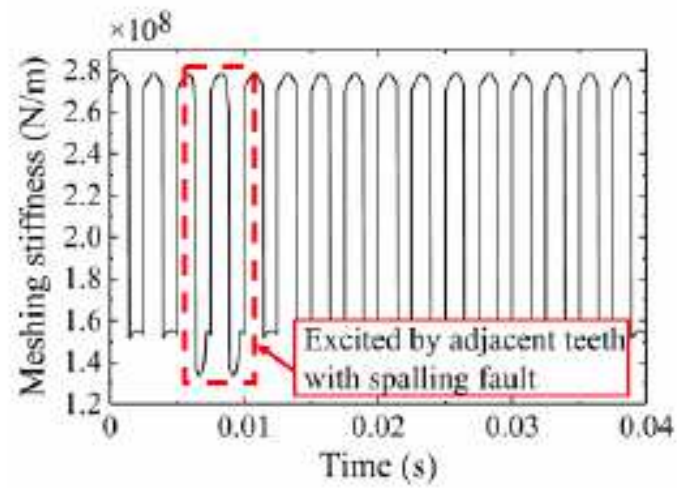

(b) Meshing stiffness curves 
Figure 9

Status of tooth surface and mesh ing stiffness curves (adjacent teeth with spalling fault)
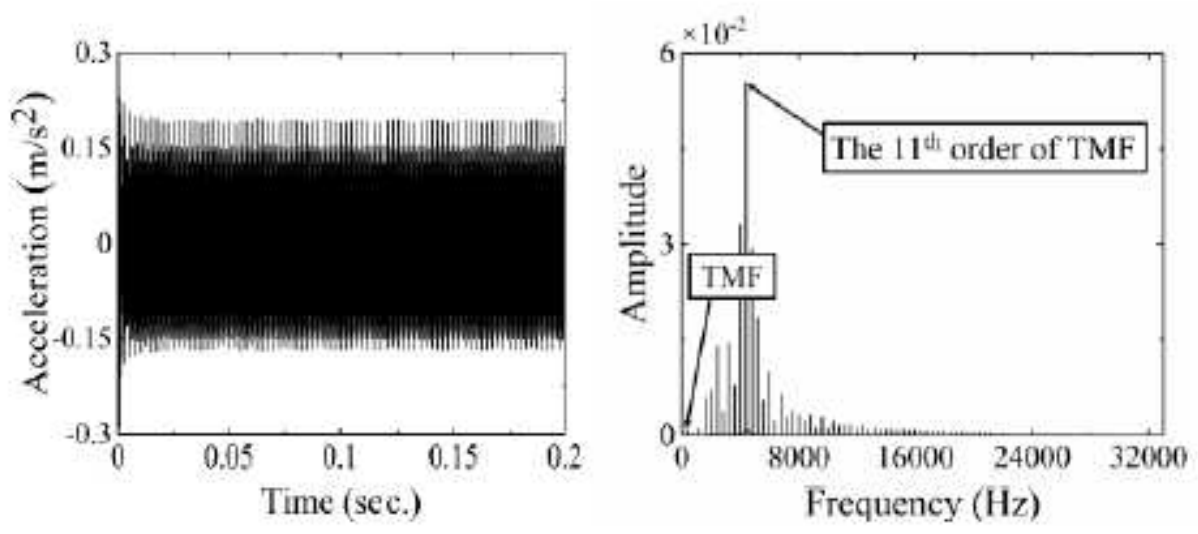

(a) Simulated signal

(b) Simulated frequency signal
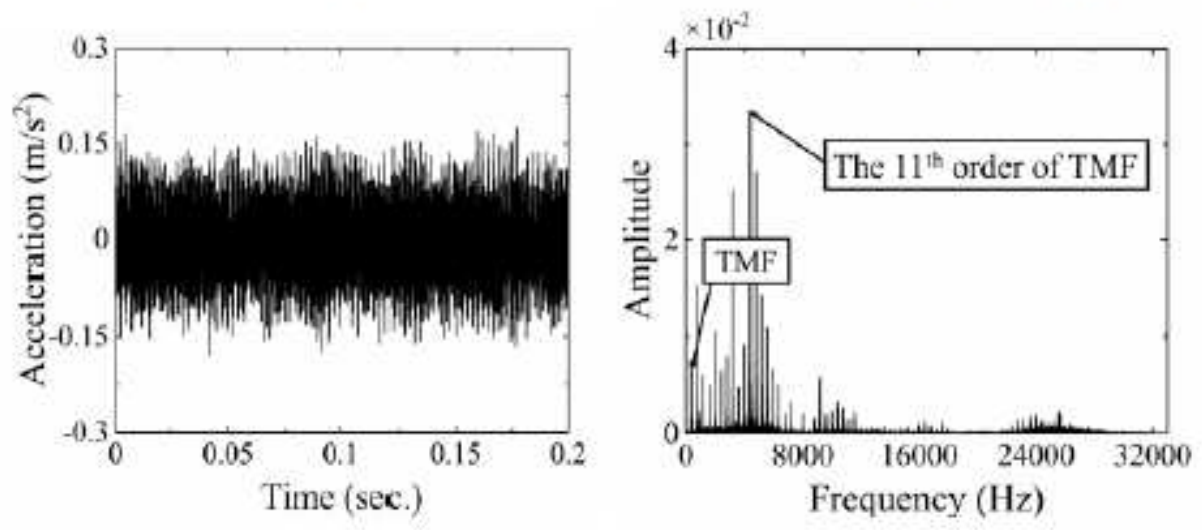

$\begin{array}{ll}\text { (c) Experimental signal } & \text { (d) Experimental frequency signal }\end{array}$

Figure 10

The simulated and experimental signal in the meshing line direction (healthy pinion) 


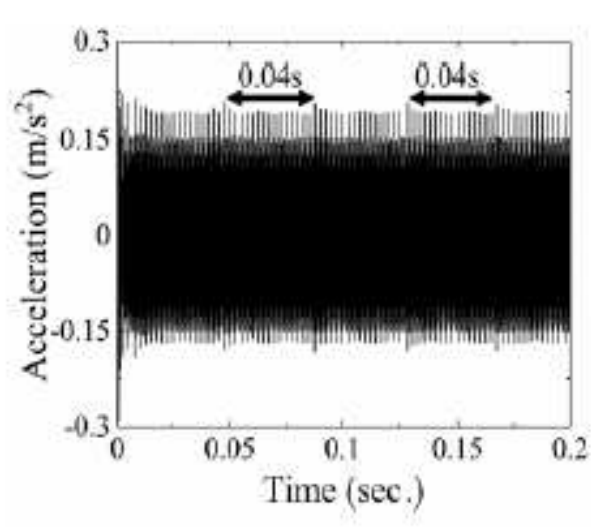

(a) Simulated signal

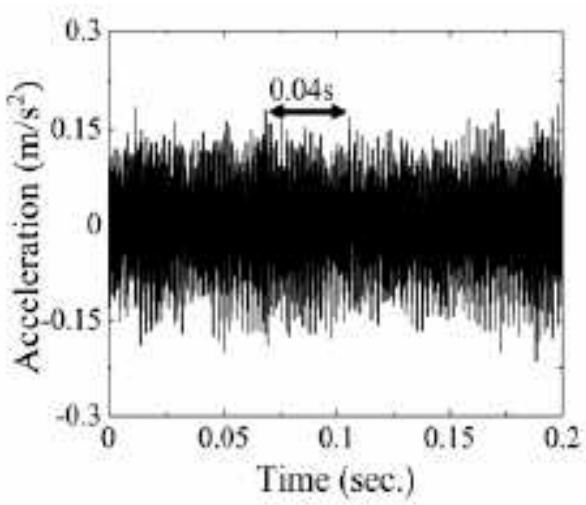

(c) Experimental signal

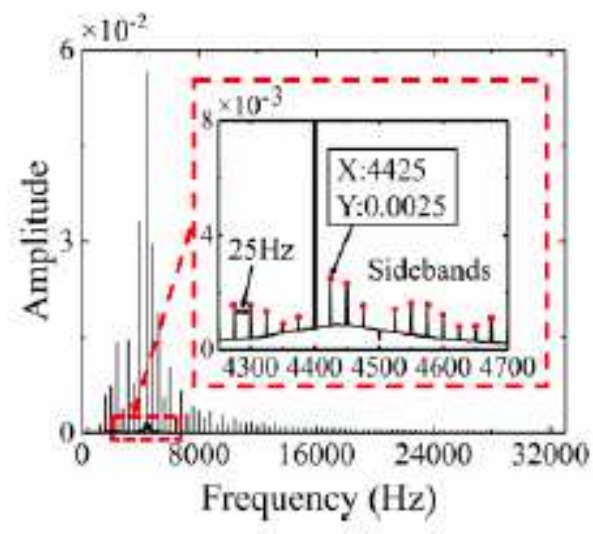

(b) Simulated frequency signal

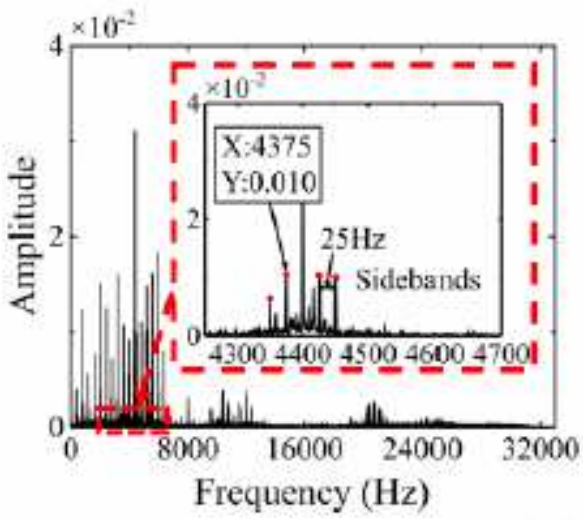

(d) Experimental frequency signal

\section{Figure 11}

The simulated and experimental signal in the meshing line direction (single tooth with spalling fault) 


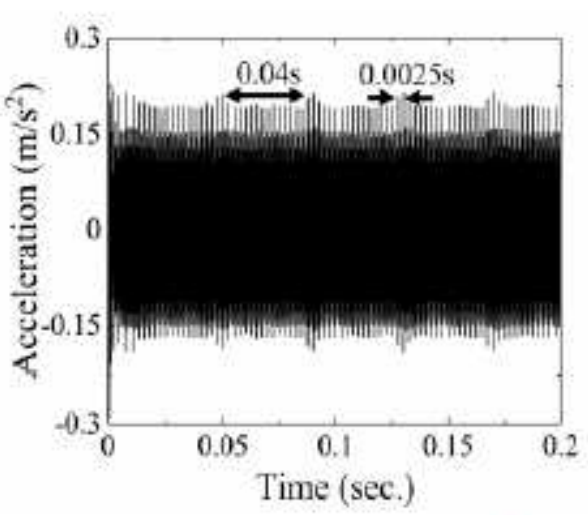

(a) Simulated signal

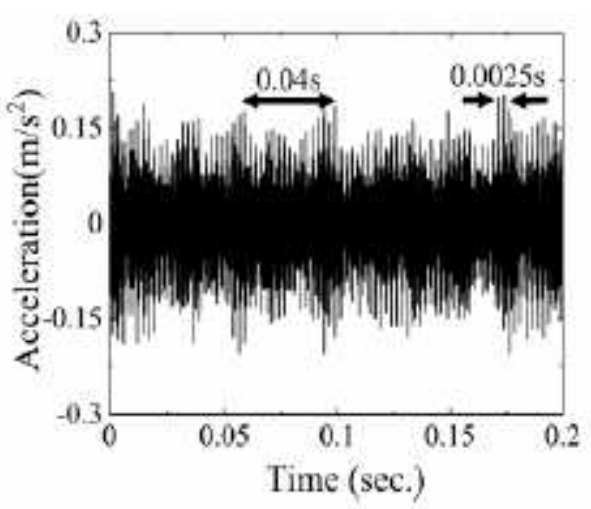

(c) Experimental signal

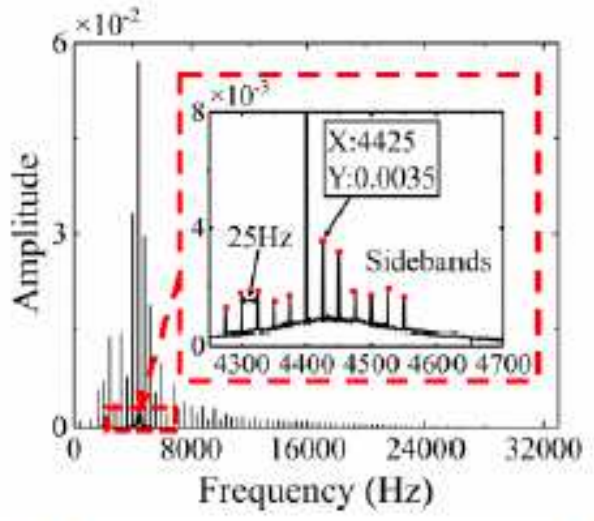

(b) Simulated frequency signal

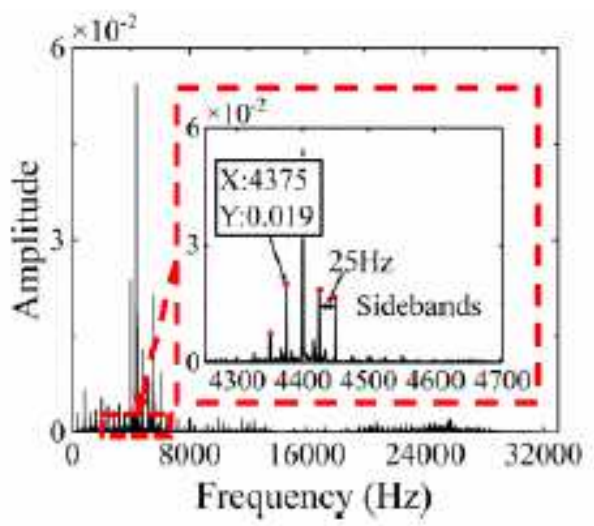

(d) Experimental frequency signal

\section{Figure 12}

The simulated and experimental signal along the line of action (adjacent teeth spalling fault)

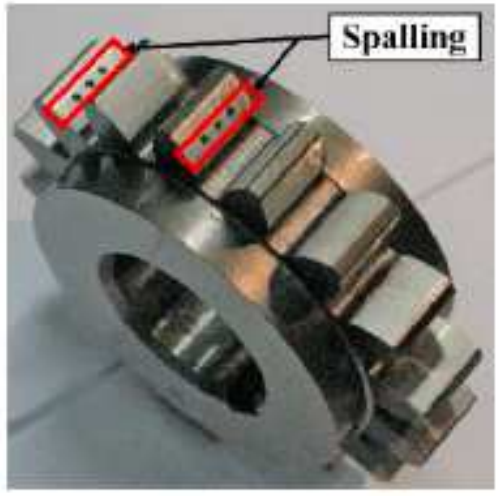

(a) Status of tooth surface

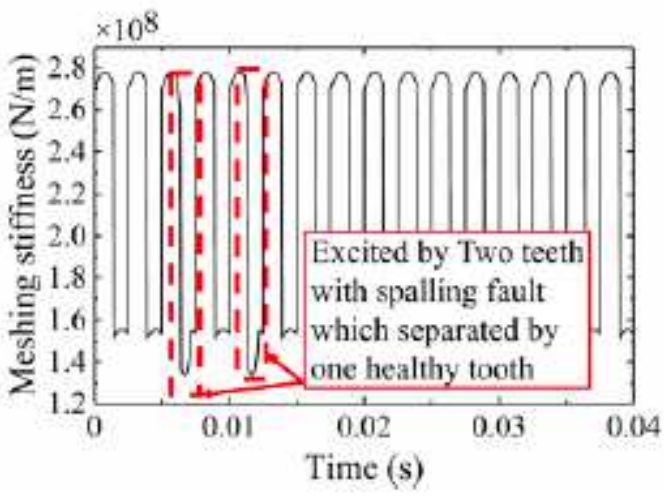

(b) Meshing stiffness curves

\section{Figure 13}

Status of tooth surface and meshing stiffness curves (two teeth with spalling fault which separated by one healthy tooth) 


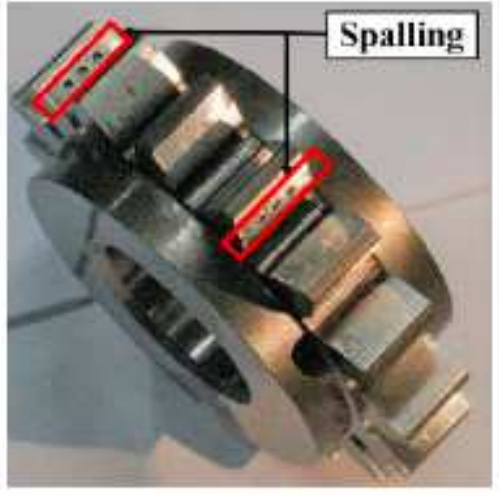

(a) Status of tooth surface

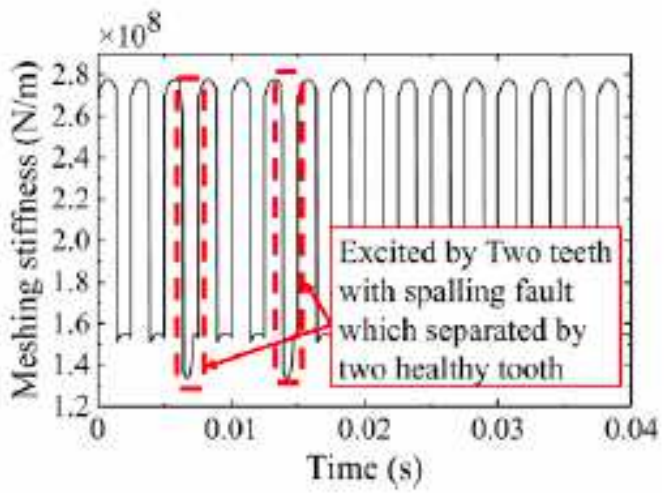

(b) Meshing stiffness curves

\section{Figure 14}

Status of tooth surface and meshing stiffness curves (two teeth with spalling fault which separated by two healthy teeth)

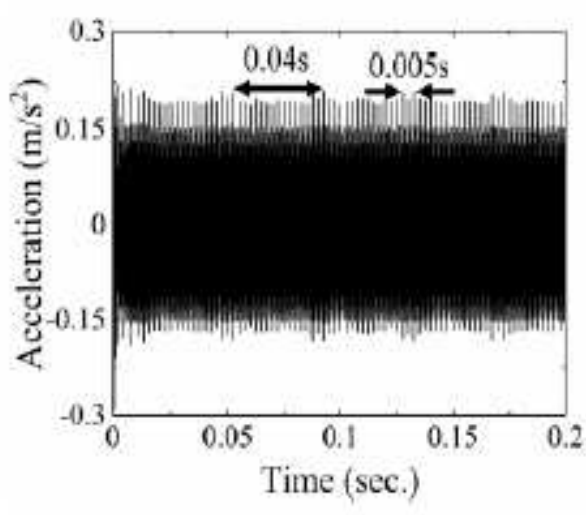

(a) Simulated signal

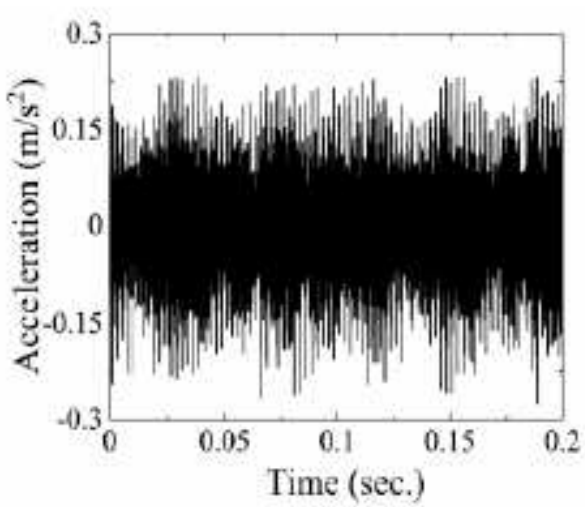

(c) Experimental signal

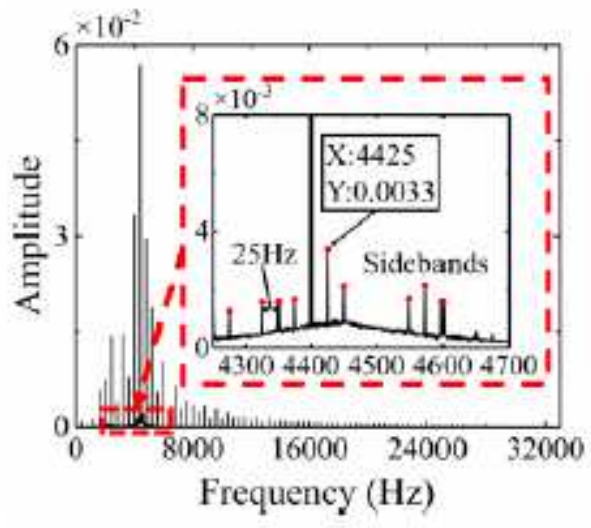

(b) Simulated frequency signal

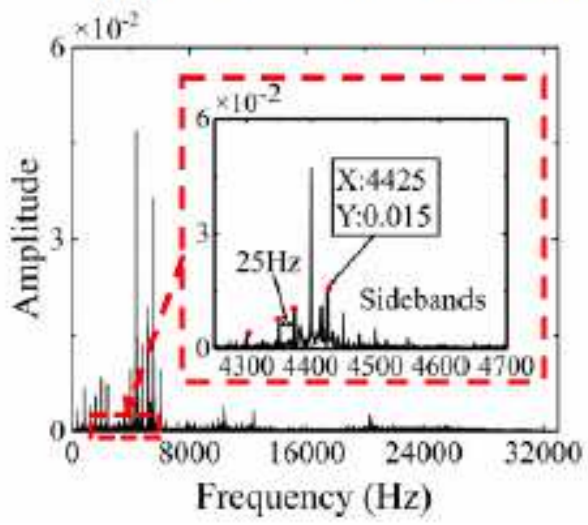

(d) Experimental frequency signal

\section{Figure 15}

The simulated and experimental signal in the meshing line direction (two teeth with spalling fault which separated by one healthy tooth) 


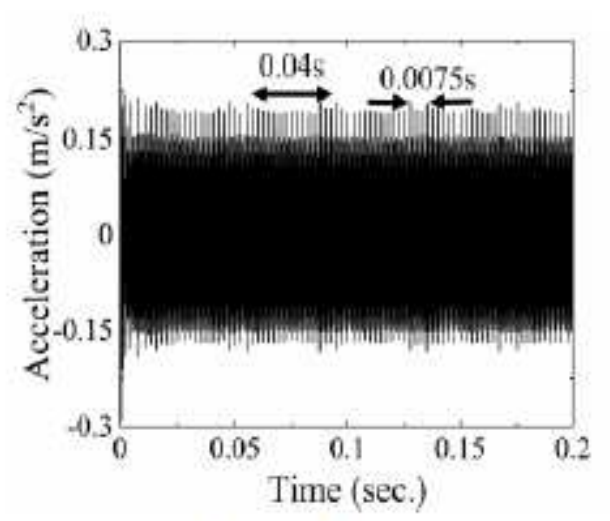

(a) Simulated signal

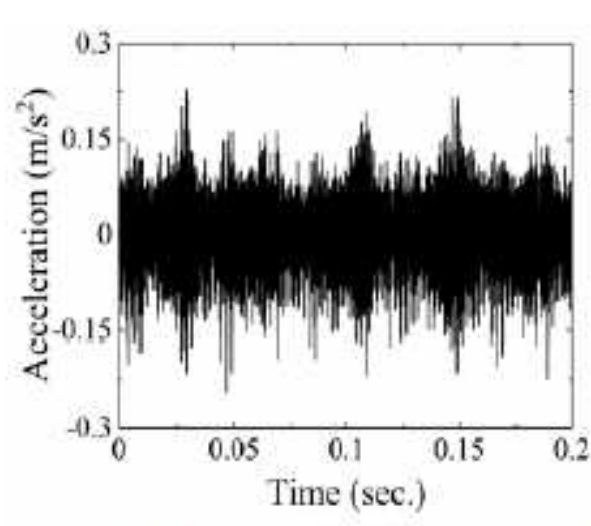

(c) Experimental signal

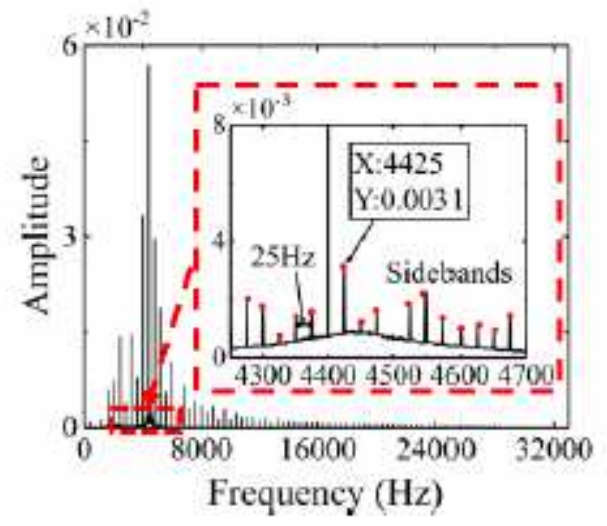

(b) Simulated frequency signal

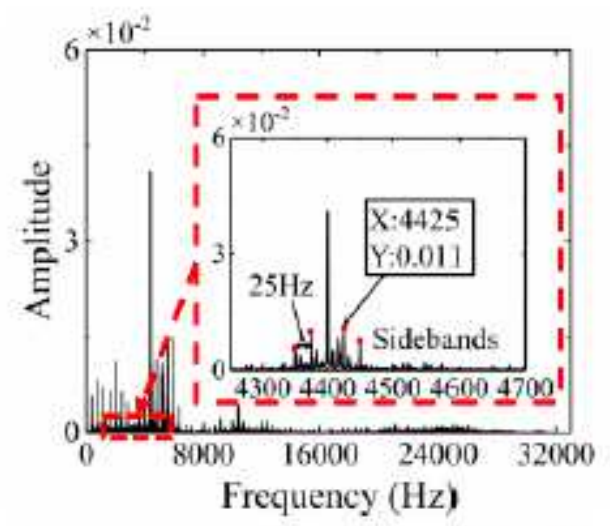

(d) Experimental frequency signal

\section{Figure 16}

The simulated and experimental signal in the meshing line direction (two teeth with spalling fault which separated by two healthy teeth) 


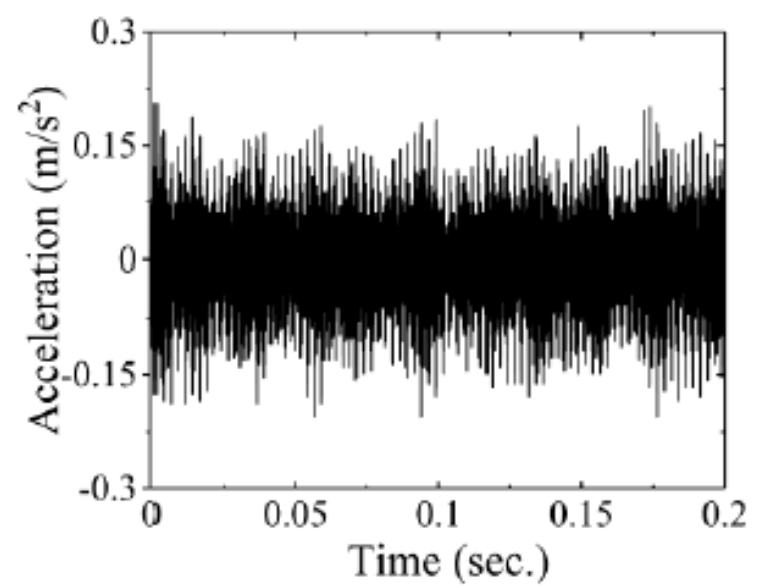

(a) $1500 \mathrm{r} / \mathrm{min}$

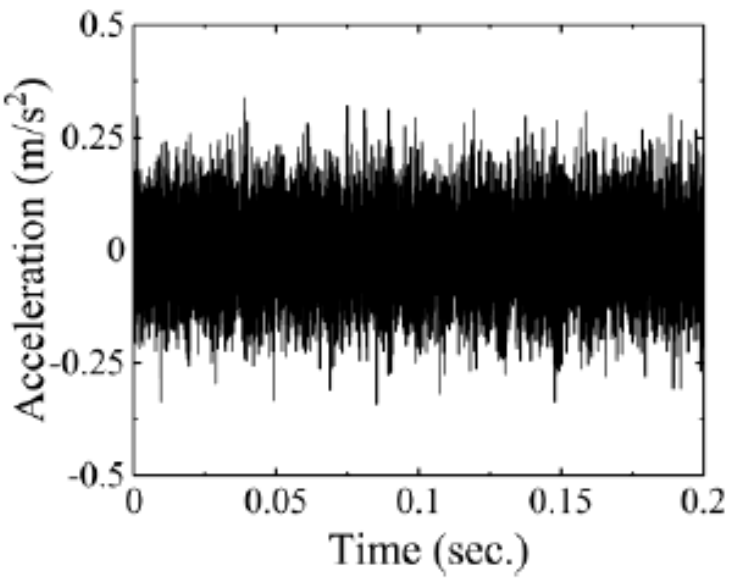

(b) $3000 \mathrm{r} / \mathrm{min}$

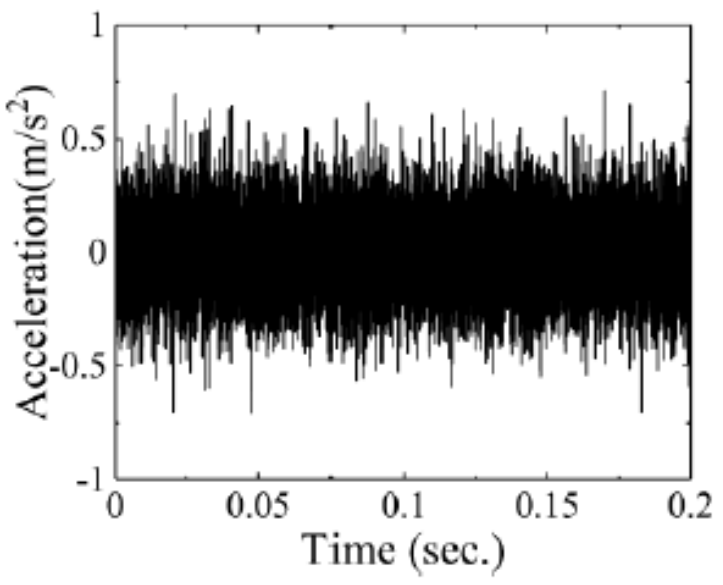

(c) $4500 \mathrm{r} / \mathrm{min}$

Figure 17

The experimental time-domain signal in the direction of meshing line under three pinion rotational speed (pinion with adjacent spalling teeth) 


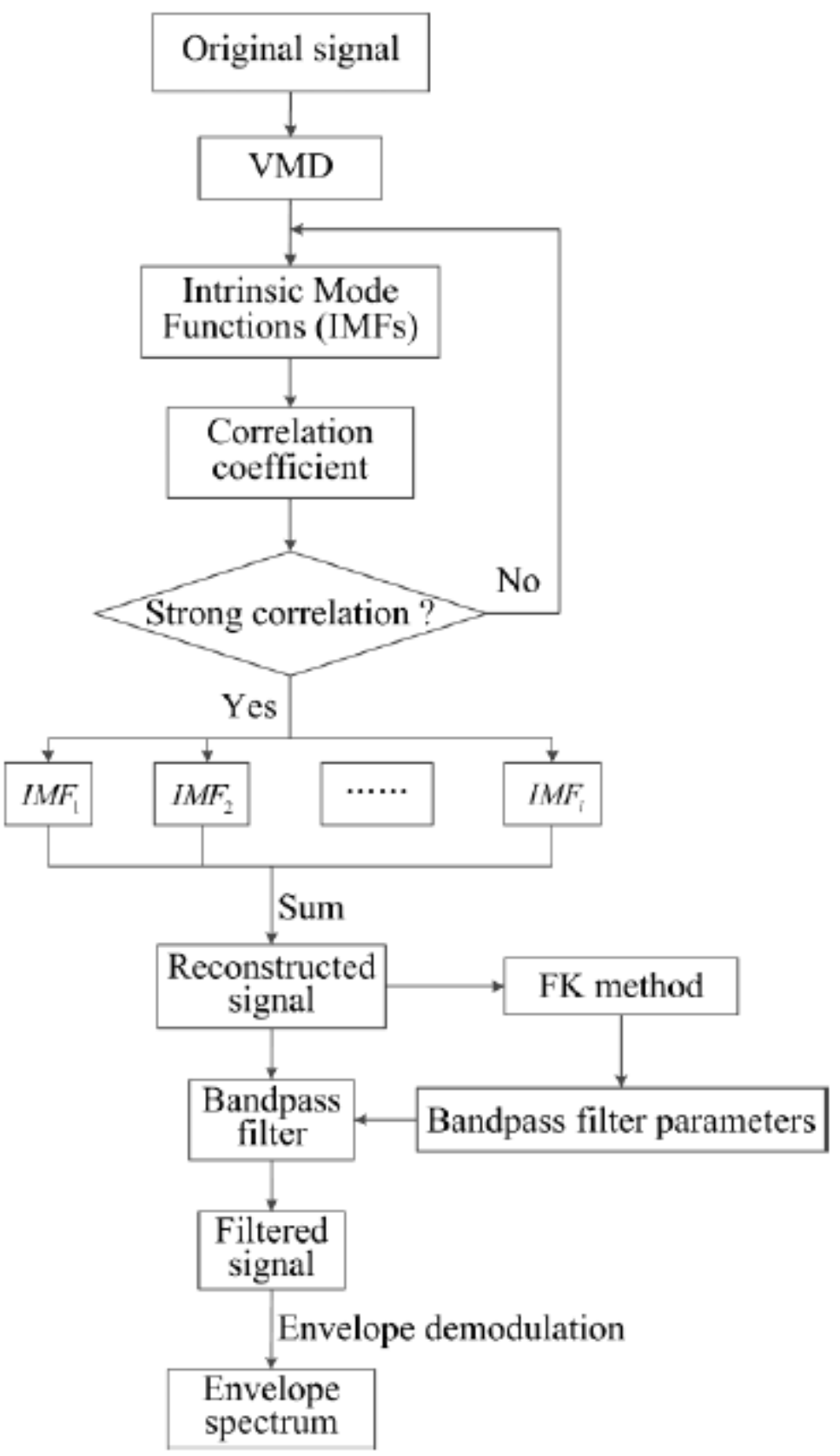

Figure 18

The procedure of the feature extraction method based on the VMD and the FK 

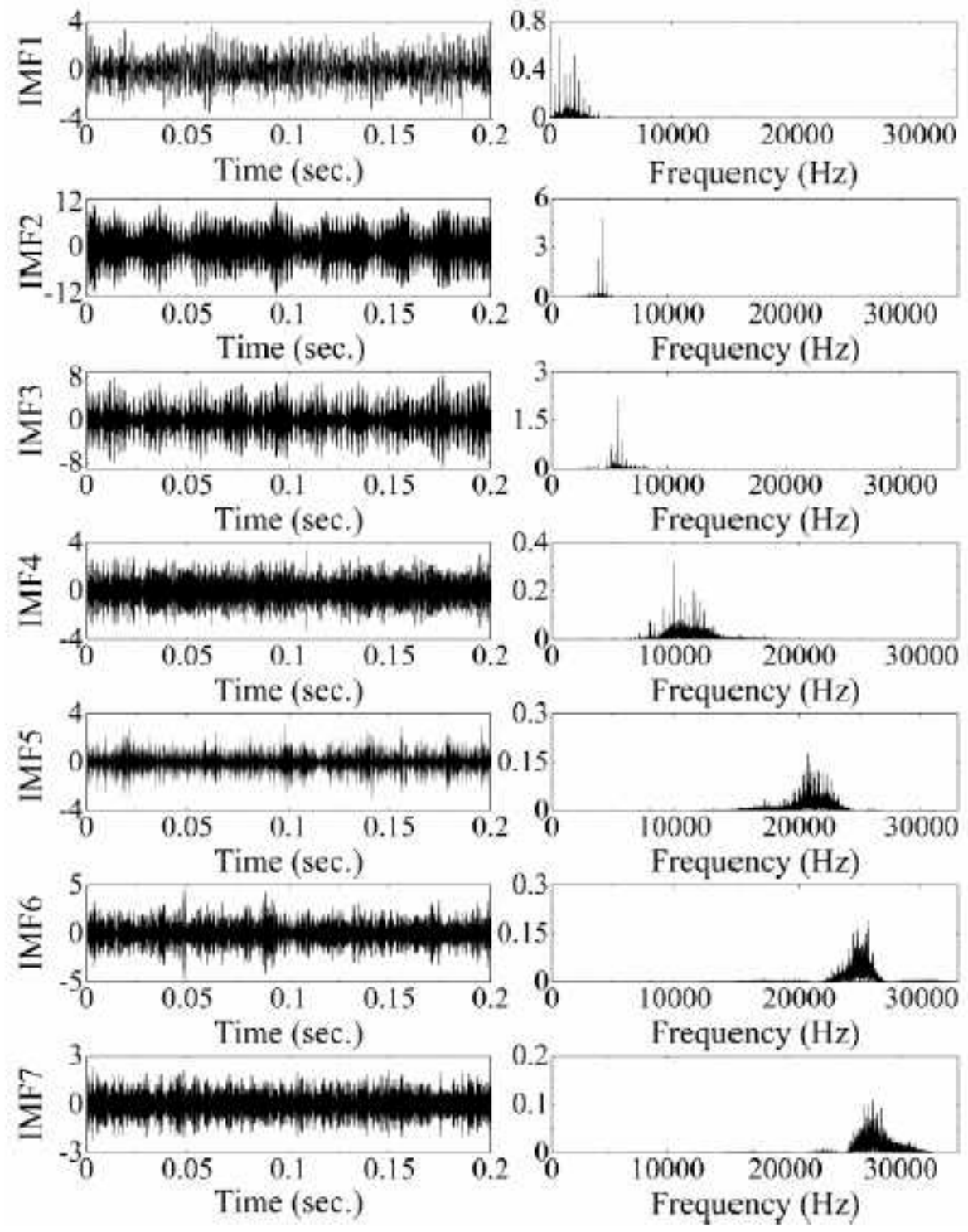

Figure 19

Results of decomposition based on the VMD for the experimental signal 


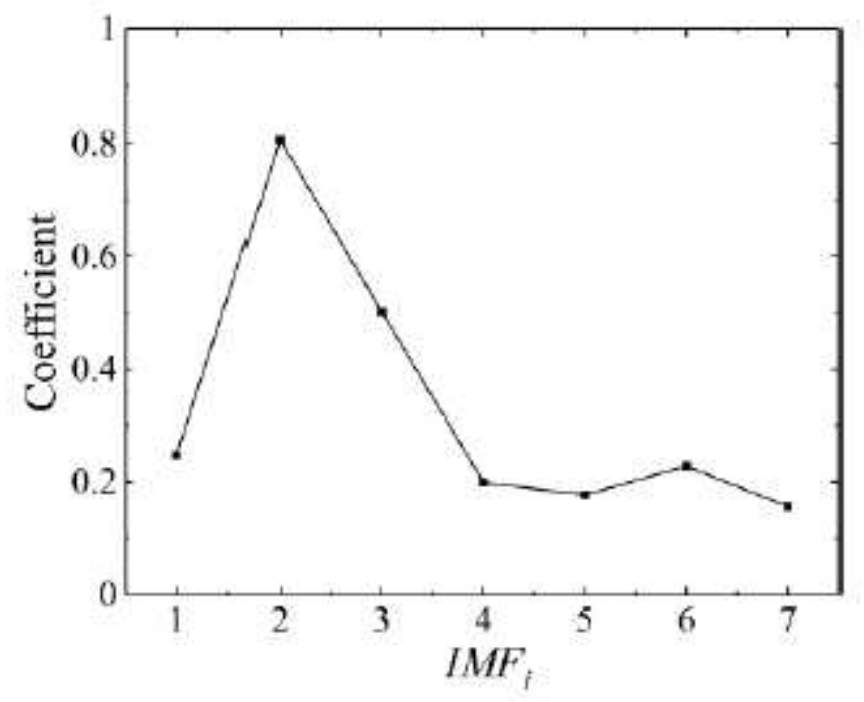

Figure 20

The correlation coefficient between each IMF and the original experimental signal

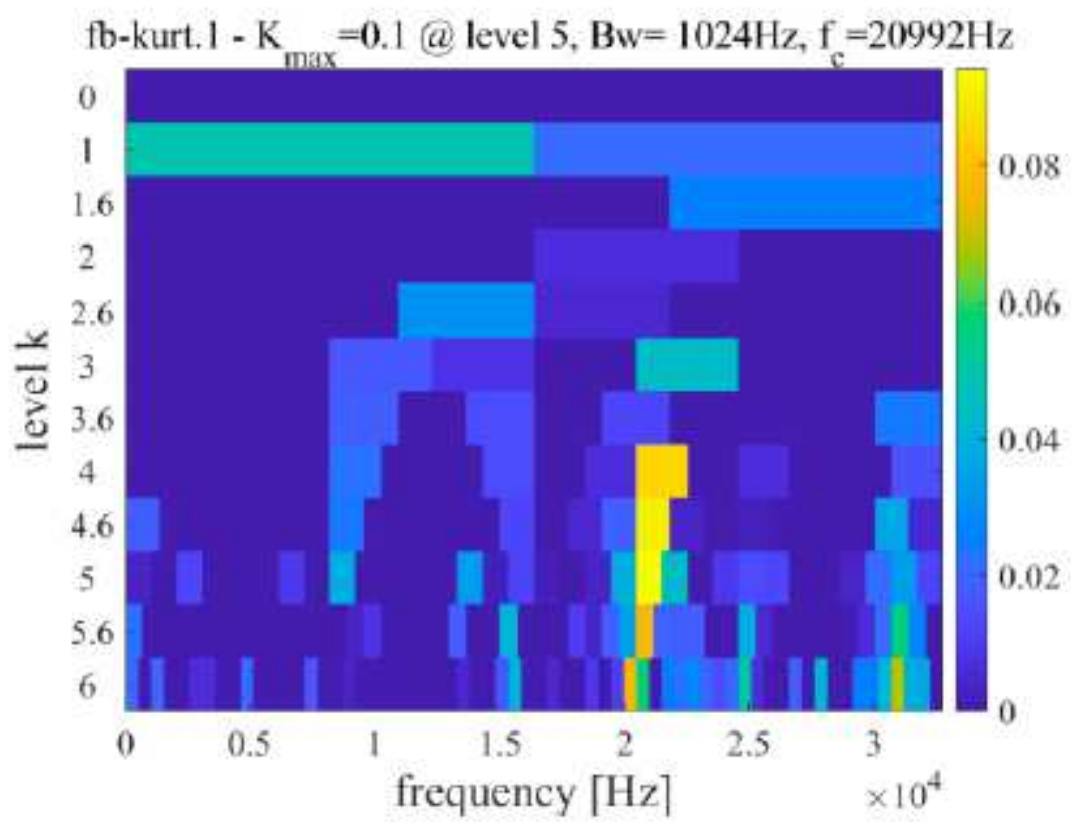

Figure 21

The Kurtogram of the reconstructed signal 


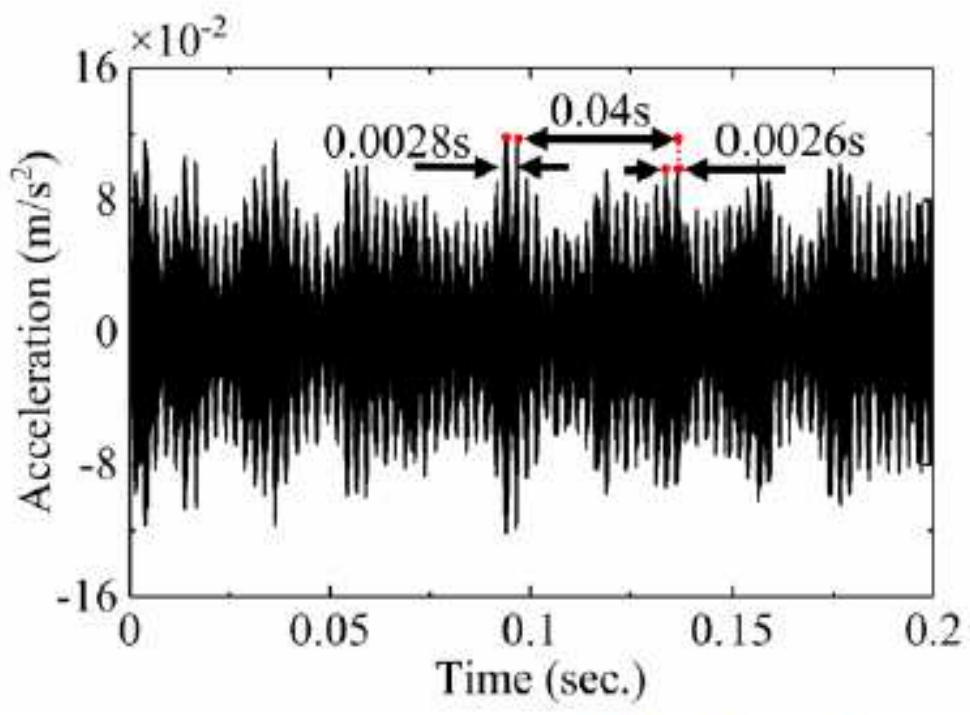

(a)The time-domain filtered signal

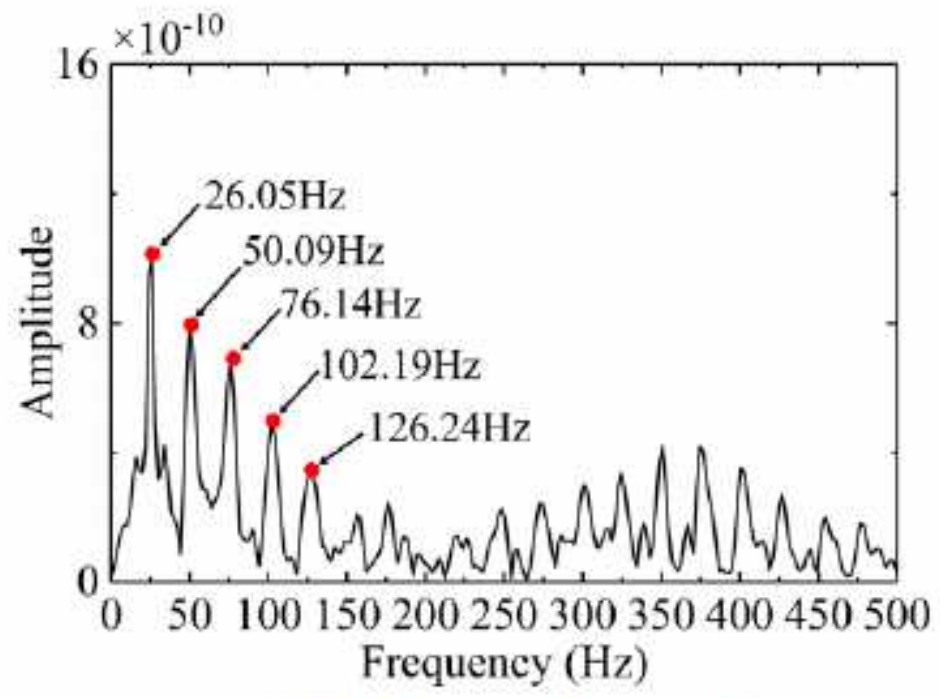

(b)The envelope spectrum

Figure 22

The results of the proposed method based on VMD and FK for the gear system experimental signal with double-teeth spalling fault spalling fault 


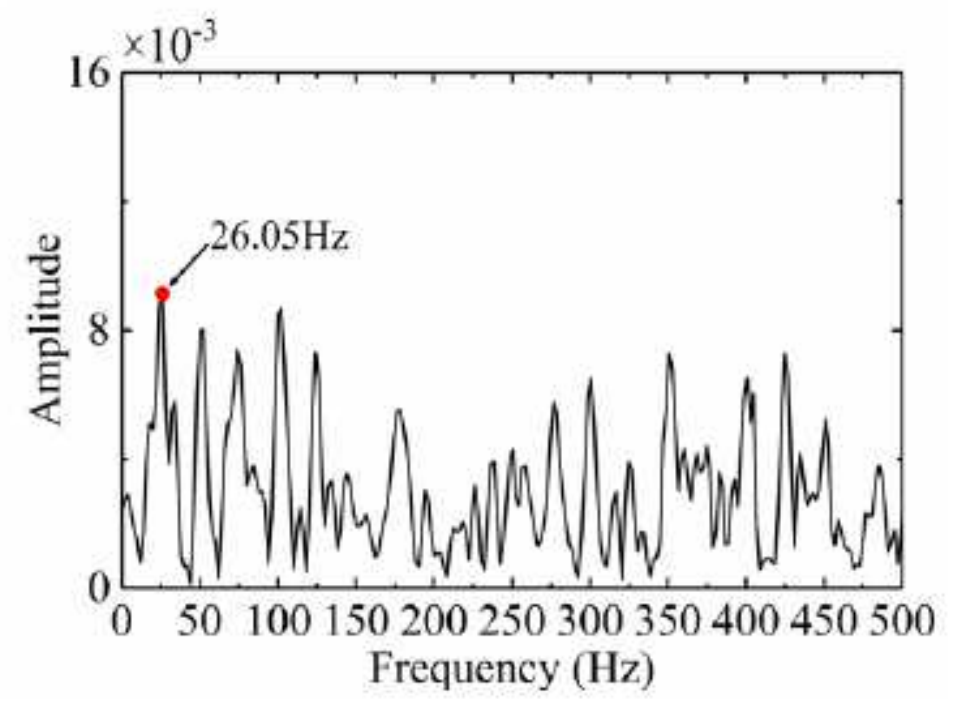

Figure 23

The envelop spectrum of the original FK method for the gear system experimental signal with doubleteeth spalling fault

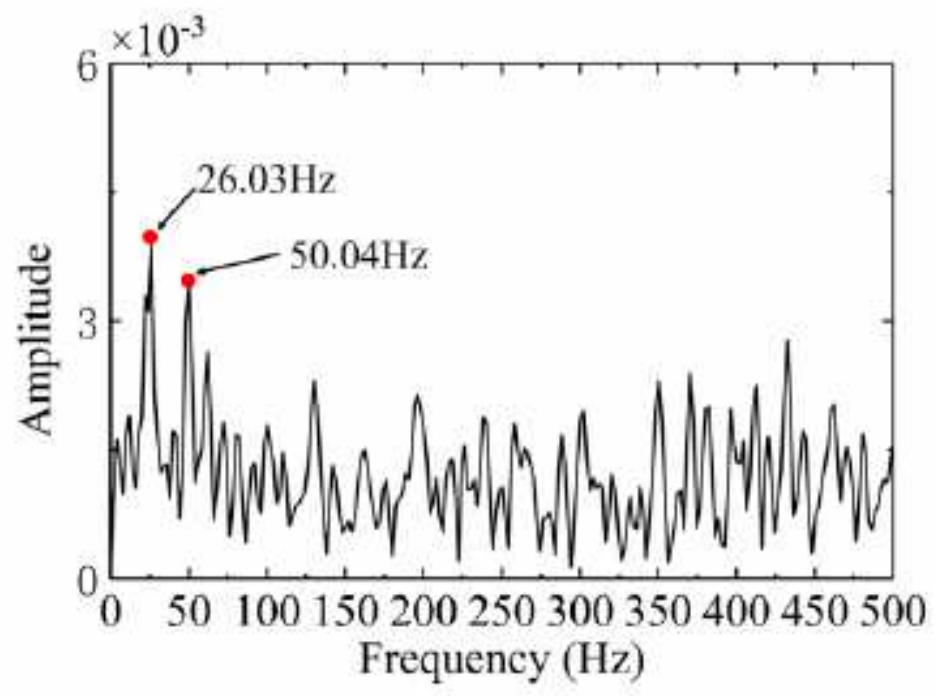

Figure 24

The envelop spectrum of the method combined with the EMD and the FK for the gear system experimental signal with double-teeth spalling fault 